\title{
Mechanisms of splicing substrate definition by modulation of pre-mRNA 3D structural scaffolds
}

\author{
Kaushik Saha* and Gourisankar Ghosh* \\ Department of Chemistry and Biochemistry, University of California San Diego, 9500 Gilman \\ Drive, La Jolla, CA 92093-0375 \\ *Correspondence: gghosh@ucsd.edu, kasaha@ucsd.edu
}

\begin{abstract}
Coordination of different serine-arginine-rich (SR) proteins - a class of critical splicing activators - facilitates recognition of the highly degenerate mammalian splice signal sequences against the background sequences. Yet, the mechanistic details of their actions remain unclear. Here we show that cooperative binding of SR proteins to exonic and intronic motifs remodels the premRNA 3D structural scaffold. The scaffold generated by the pre-mRNA-specific combinations of different SR proteins in an appropriate stoichiometry is recognized by U1 snRNP - the 5' splice site recognizing factor. A large excess of U1 snRNP particles displaces majority of the bound SR protein molecules from the remodeled pre-mRNA. A higher than optimal stoichiometry of SR proteins occludes the binding sites on the pre-mRNA, raising the U1 snRNP levels required for SR protein displacement and potentially impeding spliceosome assembly. This novel splicingrelated function of U1 snRNP is important for distinguishing the substrate and the non-substrate by U2AF65 - the primary 3' splice site-recognizing factor. Overall, this work elucidates early regulatory steps of mammalian splicing substrate definition by SR proteins.
\end{abstract}




\section{INTRODUCTION}

The early spliceosome defines the mammalian splicing substrate by recognizing four major splice signals namely the $5^{\prime}$ and $3^{\prime}$ splice sites (SS) located at the $5^{\prime}$ - and $3^{\prime}$-ends of the intron as well as branch-point site (BS) and polypyrimidine tract (PPT) near the 3'SS. U1 small nuclear ribonucleoprotein (U1 snRNP), U2 auxiliary factor 35 (U2AF35), splicing factor 1 (SF1), and U2 auxiliary factor 65 (U2AF65), respectively, are reported to recognize them (1). U2AF65 is considered to be the primary factor that defines the 3 'SS $(2,3)$. These recognition events, however, are not governed just by the splice signal sequences but a combination of a variety of features embedded within the pre-mRNA strand, collectively known as the 'splicing code' (4). The sequence of the splice signals, the pre-mRNA secondary structure $(5,6)$, the splicing regulatory element (SREs) (7), and the pre-mRNA 3D structural scaffold (8) may contribute to the splicing code of a pre-mRNA. SREs recruit from a large repertoire of RNA binding proteins (RBP) for managing the recognition-potential of a splice signal (7). Serine-arginine-rich (SR) proteins are key players in regulation of early spliceosome assembly and deregulation of the process causes widespread splicing changes leading to a wide range of diseases (9-17). Accurate correlation of their binding to the cognate SREs within the pre-mRNA with the splicing outcome is critical for elucidation of the mammalian 'splicing code (18). Earlier we showed that strandedness of exonic segments immediately upstream of the 5 'SS critically regulate binding of SR proteins for pre-mRNA structural remodeling (6). We observed that multiple copies of a specific SR protein could remodel the 3D structural scaffold of the pre-mRNA generating a recognizable substrate for the early spliceosomal components (8). The success of this remodeling event by the specific SR protein also could depend on the initial 3D structure of the pre-mRNA. That is, just the presence of the binding motifs was not sufficient to exert the specific functions of the SR proteins. However, splicing regulation involves a complex interplay among 
multiple SR proteins bound at both exons and introns, which could be synergistic, antagonistic, and/or compensatory (18). Currently, the mechanistic detail of this collaboration remains unclear. Interactions of SR proteins within the assembled early spliceosome is well-characterized (19). There a single molecule of a specific type of SR protein bound at an exonic splicing enhancer (ESE) directly interacts with an early spliceosomal component bound at its cognate splice signal sequence. However, this does not highlight the collaborative nature of SR proteinmediated regulation of early recognition of the splice sites. Additionally, early splicing substrate definition by SR proteins may also occur without a detectable interaction between the SR protein and the early spliceosomal component and could primarily be mediated by SR proteinmediated pre-mRNA structural remodeling (8). Thus, mechanistic details of the collaborative actions of the SR proteins for the early recognition of a specific SS still remains unclear (20).

There are also additional aspects of SR protein actions that are mechanistically unclear. SR proteins may repress splicing by binding to intronic motifs (18) through an as yet uncharacterized mechanism. Moreover, SR proteins may bind the pre-mRNAs in multiple copies prior to assembly of the early spliceosome $(6,19)$ but only one or two are reported to remain bound within the assembled early spliceosome (19); their displacement mechanisms are not yet characterized.

In the current work, we characterized the regulatory collaboration of three SR proteins SRSF1, SRSF2, and SRSF5 - for recruitment of U1 snRNP to full-length pre-mRNA substrates. We primarily used human $\beta$-globin IVS1 for dissecting these functions since each of these SR proteins promote the splicing of this substrate $(21)$ and the technique for resolving the premRNA complexes could clearly distinguish the SR protein-dependent and SR proteinindependent U1 snRNP- $\beta$-globin complexes. Our data highlights the principles of regulatory collaboration among SR proteins, identifies a novel function of U1 snRNP critical for splicing 
bioRxiv preprint doi: https://doi.org/10.1101/2021.12.01.470860; this version posted December 9, 2021. The copyright holder for this preprint (which was not certified by peer review) is the author/funder. All rights reserved. No reuse allowed without permission.

substrate definition, and provides a greater understanding of the 3D structural scaffold and its modulation for splicing substrate definition. 


\section{METHODS}

\section{Cloning, protein expression, and in vitro reconstitution and purification of U1 snRNP}

Different subunits of U1 snRNP and the SR proteins were expressed and purified as described before (8). Unless otherwise indicated, U1 snRNP was reconstituted and purified with all fulllength proteins except U1-70k, which was truncated (1-215 amino acids) as described before (8)

\section{RNA constructs}

B-globin and AdML RNA were as described before (6). 14-nt long Ron ESE (5'UGGCGGAGGAAGCA-3') sequence and $\beta$-globin 5'SS (5'-GGGCAGGUUGGUAU-3') were described before $(8,24)$..

\section{Electrophoretic mobility shift assay (EMSA)}

EMSA with full-length pre-mRNA and 14-nt long RNAs were carried out with 4\% (89:1) and 6\% (80:1) native polyacrylamide gels, respectively, as described before (8). Antibody super-shift was carried out with anti-SNRPC antibody (Abcam, ab157116) and anti-SRSF1 antibody (Life Technologies, 32-4500). After the formation of complexes as described above, $0.25 \mu \mathrm{g}$ antibody was added to the $15 \mu$ reaction, incubated at $30{ }^{\circ} \mathrm{C}$ for $5 \mathrm{~min}$ and resolved on polyacrylamide gels.

In vitro selective 2' hydroxyl acylation followed by primer extension by mutational profiling (SHAPE-MaP)

All SHAPE assays were carried out with samples treated with $4 \mathrm{mM} \mathrm{N}$-Methylisatoic anhydride (NMIA, Millipore-Sigma). For SHAPE assay with $\beta$-globin, first $800 \mathrm{nM}$ RNA was denatured at $95{ }^{\circ} \mathrm{C}$ for $3 \mathrm{~min}$ in $50 \mathrm{mM} \mathrm{NaCl}$ and $5 \mathrm{mM}$ EDTA pH 8.0 and snap-cooled on ice. After neutralizing EDTA with $\mathrm{MgCl}_{2}, 100 \mathrm{nM}$ (final concentration) RNA was mixed in $1 \mathrm{ml}$ reaction containing $20 \mathrm{mM}$ HEPES pH 7.5, $250 \mathrm{mM} \mathrm{NaCl}, 2 \mathrm{mM} \mathrm{MgCl}_{2}, 0.5 \mathrm{M}$ urea, $0.3 \%$ polyvinyl alcohol (P-8136, Sigma), and $1 \mathrm{mM}$ 2-mercaptoethanol along with the indicated level of SR 
proteins or SR protein storage buffer and incubated at $30^{\circ} \mathrm{C}$ for $5 \mathrm{~min}$. Then the solution was transferred to a fresh tube containing $33.3 \mu \mathrm{l} 120 \mathrm{mM}$ NMIA or DMSO (vehicle), mixed immediately, and incubated at $30{ }^{\circ} \mathrm{C}$ for $15 \mathrm{~min}$ and then at room temperature for $15 \mathrm{~min}$. Thereafter, $9 \mathrm{M}$ urea is added to the reaction up to the final concentration of $2 \mathrm{M}$ and then the reaction was treated with $0.5 \mathrm{mg} / \mathrm{ml}$ (final concentration) proteinase $\mathrm{K}(\mathrm{NEB})$ at $37^{\circ} \mathrm{C}$ for $30 \mathrm{~min}$. Next, RNA was ethanol-precipitated with $300 \mathrm{mM}$ sodium acetate $\mathrm{pH} 5.2$, the pellet was dissolved in $50 \mu \mathrm{l}$ water, and then the RNA was purified by Monarch RNA cleanup kit (New England Biolabs).

For U1 snRNP binding to $\beta$-globin+SRSF1-RBD complex, a very high concentration of U1 snRNP had to be added to the reaction. Raising U1 snRNP to such a concentration was difficult. Additionally, the presence of such a high concentration of U1 snRNP in the reaction might affect overall SHAPE reactivity and hence, it was necessary to purify the complex. However, purifying this complex by amylose pull-down was inefficient due to weaker binding of U1 snRNP to $\beta$-globin compared to AdML. Therefore, it was not possible to carry out SHAPE reaction on the ternary complex of $\beta$-globin. For SHAPE assay with $A d M L, 500 \mathrm{nM}$ refolded RNA with 3X MS2-binding loops at the 3 ' end was first bound to 1500 nM MBP-MS2 protein for $15 \min$ at $30{ }^{\circ} \mathrm{C}$. Then the RNA was added to $250 \mu \mathrm{l}$ reaction mixture with the same buffer composition as with $\beta$-globin at a final concentration of $100 \mathrm{nM}$, was mixed with $300 \mathrm{nM}$ U1 snRNP or 500 nM SRSF1 (full-length SRSF1 with all serine residues in its RS domain replaced with glutamate) or both, and was incubated at $30^{\circ} \mathrm{C}$ for $5 \mathrm{~min}$. Then complexes were bound to $15 \mu \mathrm{l}$ amylose agarose (NEB) by rotating the tube at $4{ }^{\circ} \mathrm{C}$ for $30 \mathrm{~min}$. The resin was washed with chilled buffer (20 mM HEPES $\mathrm{pH}$ 7.5, $250 \mathrm{mM} \mathrm{NaCl}, 2 \mathrm{mM} \mathrm{MgCl}$, $1 \mathrm{mM}$ 2mercaptoethanol) and then eluted in $500 \mu \mathrm{l}$ of this buffer containing $20 \mathrm{mM}$ maltose for rotating the tube at $4{ }^{\circ} \mathrm{C}$ for 2 hours. Then $250 \mu$ solution was transferred to $8.33 \mu \mathrm{l} 120 \mathrm{mM}$ NMIA or equal volume of DMSO (vehicle), mixed gently, and incubated at $16^{\circ} \mathrm{C}$ for 1 hour. The solution 
was then treated with $0.5 \mathrm{mg} / \mathrm{ml}$ proteinase $\mathrm{K}$ in the presence of added $2 \mathrm{M}$ urea at $37^{\circ} \mathrm{C}$ for 30 min and the RNA was ethanol-precipitated followed by RNA cleanup with Monarch RNA cleanup kit. Preparation of denatured control, library preparation, deep sequencing, and data processing were carried out as described before (8). SHAPE-derived secondary structure models were obtained using RNAstructure software (54).

SHAPE differentials were calculated by determining fold-changes in SHAPE reactivity of a pre-mRNA upon binding to a splicing factor compared to the protein-free pre-mRNA. Chemical reactions of flexible nucleotides with structure probing reagents are sensitive to local chemical environment and hence, fold-changes likely reflect a more meaningful information regarding structural alteration of the RNA. For calculating fold-changes, all nucleotides with zero or negative SHAPE reactivity values were considered to be 0.0001 .

\section{Detection of RNA-protein interactions by RNP-MaP}

It was not possible to carry out RNP-MaP on $\beta$-globin+SRSF1-RBD+U1 snRNP complex because it was necessary to purify the complexes by amylose pull-down before carrying out RNP-MaP (also see the method for SHAPE). This is because the complexes are assembled in the presence of $0.5 \mathrm{M}$ urea and $\mathrm{U} 1 \mathrm{snRNP}$ is stored in a buffer containing arginine- $\mathrm{HCl}$ and glutamate-KOH, which would interfere with the SDA crosslinking reagent used for RNP-MaP since SDA reacts with primary amines. Thus, purified complexes were needed for RNP-MaP analysis. Since we could only purify $A d M L$ complexes efficiently by amylose pull-down, we carried out RNP-MaP with AdML only. RNP-MaP for detection of protein interaction sites on an RNA was carried out as described before (55). AdML complexes were assembled and purified as in SHAPE. The complex was eluted by rotating the tubes for 2 hours at $4{ }^{\circ} \mathrm{C}$ in elution buffer containing 4 mM SDA (NHS-diazirine, succinimidyl 4,4'-azipentanoate, Thermo Fisher Scientific, diluted from $100 \mathrm{mM}$ stock in DMSO) or equivalent concentration of DMSO. Then the resin was 
removed by gentle centrifugation, the liquid was transferred to a 24-well plate on ice and was exposed to $3 \mathrm{~J} / \mathrm{cm}^{2}$ of $365 \mathrm{~nm}$ light in UVP 1000CL (Analytik Jena) crosslinker. The proteins were then digested in the presence of $1.5 \%$ SDS, $20 \mathrm{mM}$ EDTA, and $0.5 \mathrm{mg} / \mathrm{ml}$ proteinase $\mathrm{K}$ for 2 hours at $37{ }^{\circ} \mathrm{C}$ and the RNA was purified by phenol:chloroform extraction and ethanol precipitation. The RNA pellet was dissolved in $50 \mu \mathrm{l}$ water and was further purified by Monarch RNA cleanup kit. The reverse transcription reaction was carried out as described before (55). The library preparation and deep sequencing were carried out as in SHAPE. Analyses of RNP sites and RNP reactivities were carried out as described before (55).

\section{In vivo DMS-MaP-seq}

HeLa cells grown in six-well plate were co-transfected with $5 \mathrm{nmol}$ of U1 snRNA antisense morpholino oligonucleotide (56) and $1 \mu \mathrm{g} \beta$-globin minigene plasmid and were incubated for 18 hours. Cells were then treated with $2 \%$ dimethyl sulfate (DMS, Millipore Sigma) in $2 \mathrm{ml}$ incomplete DMEM medium and incubated at $37^{\circ} \mathrm{C}$ for $5 \mathrm{~min}$. Then the medium was aspired out and cells were washed twice with $30 \%$ 2-mercaptoethanol in PBS. Total RNA was then extracted with Trizol (Thermo Fisher Scientific) with DNase treatment following manufacturer's instructions. Reverse transcription was carried out with TGIRT III reverse transcriptase (Ingex) as described previously (31). Due to modification of the RNA, which are substituted by mutations during reverse transcription, the primers used for sequencing library preparation lost significant complementarity to the cDNA. The weak annealing prevented amplification of the 450-nt long $\beta$-globin pre-mRNA with end-to-end amplification. Therefore, we split the cDNA into two adapted fragments for deep sequencing - 1-228 nt and 109-442 nt. DMS reactivity was obtained using DREEM (31). We used data for 1-180 nt from the $5^{\prime}$ segment and 181-442 nt from the 3 ' segment. 


\section{RESULTS}

SRSF1-RBD stabilizes the interactions between the global 3D structural scaffold of $\beta$ globin and U1 snRNP

First, we examined the specific features of $\beta$-globin that are recognized by U1 snRNP and whether SRSF1-RBD stabilizes this interaction. We titrated $10 \mathrm{pM} \beta$-globin in the presence as well as the absence of $60 \mathrm{nM}$ SRSF1-RBD with U1 snRNP (Figure 1A). With increasing concentrations of U1 snRNP, the SRSF1-RBD-bound pre-mRNA complex was downshifted and eventually formed $\sim 2$ faster migrating, apparently stable, complexes. We confirmed the presence of SRSF1-RBD as well as U1 snRNP in the final WT 'ternary' complexes by carrying out antibody super-shift with anti-SRSF1 and anti-U1-C antibodies, respectively (compare lanes $5 \& 9$ and 18 \& 19). Anti-U1C antibody also disintegrated a portion of the pre-mRNA complex likely because U1-C is important for pre-mRNA binding of U1 snRNP (22). Surprisingly, $\beta$-globin $\triangle 5$ 'SS also formed very similar complexes with U1 snRNP in the presence as well as the absence of SRSF1-RBD (Figure 1A - compare lanes 4, 5 with 13, 14 and lanes 7, 8 with 16, 17, respectively). We hypothesized that the long $3^{\prime}$ exon of $\beta$-globin (206-nt in $\beta$-globin) might provide sufficient support to U1 snRNP to stabilize it on the pre-mRNA given that the global structural landscape of a pre-mRNA could be involved in U1 snRNP recruitment (8). Therefore, we generated a $\beta$-globin construct with truncated $3^{\prime}$ exon $(\beta g-\triangle E x 2)$. EMSA indicates U1 snRNP does not exhibit distinct interaction with free $\beta g-\triangle E x 2$. In the presence of SRSF1-RBD, U1 snRNP formed distinct complexes with $\beta g-\Delta E x 2$; however, the complexes formed with $\beta g-\Delta E x 2$ $\Delta 5$ 'SS was distinctively weaker than the former (Figure $1 \mathrm{~B}$ - compare lanes 3 and 9 ). We also generated the hybridization-mutant $(E H 3+4)$ on $\beta g-\Delta E x 2$ construct, in which the critical singlestranded segments immediately upstream of the 5'SS are hybridized; this mutant does not recruit SR proteins appropriately and is defective in splicing (6). Distinct U1 snRNP-dependent 
complexes were not evident either in the presence or the absence of SRSF1-RBD (Figure 1B, lanes 15 and 17).

Since the $3^{\prime}$ exon of $\beta$-globin stabilizes U1 snRNP on $\beta$-globin, we hypothesize that U1 snRNP recognizes a 3D structural scaffold of $\beta$-globin. To examine how $\beta$-globin structure regulates U1 snRNP binding even in the absence of 5 'SS, we first examined the SHAPE reactivity of protein-free $\beta$-globin and its 5'SS-mutant and generated their SHAPE-derived secondary structure models (Supplementary Figure S1A, S1B). The largest structural differences were observed around the $5^{\prime}$ SS between $60^{\text {th }}$ to $130^{\text {th }}$ positions $\left(5^{\prime}\right.$ exon ends at the $106^{\text {th }}$ position) (Figure $1 \mathrm{C}$ ). However, strandedness of the nucleotides in the remainder of the pre-mRNA appeared somewhat similar. Moving averages (period $=6$ ) of the SHAPE reactivity of the nucleotides between $140^{\text {th }}$ and $420^{\text {th }}$ positions of both RNAs showed a decent correlation $(r=0.81, p=9.77 E-66)$. Moving averages of SHAPE reactivity of U1 snRNP-bound $\beta$-globin variants exhibited a slightly higher correlation even in a longer segment, between $120^{\text {th }}$ and $420^{\text {th }}$ position ( $r=0.83, p=1.97 \mathrm{E}-78$ ) (Figure $\left.1 \mathrm{D}\right)$. This suggests that $\beta$-globin likely has a loosely organized 3D structure and is spatially brought together by the global engagement of U1 snRNP; hence, mutation in the authentic and cryptic 5'SS did not completely disrupt the 3D structural scaffold of $\beta$-globin and U1 snRNP recruitment to it. As expected, however, $\beta$-globin $\Delta 5^{\prime}$ SS is completely defective in splicing in vivo (Supplementary Figure S1C).

We then used two 14-mer short RNA oligonucleotides ( $\beta$-globin 5'SS and Ron ESE, an SRSF1-binding ESE) to determine the stability of the $\beta$-globin:SRSF1-RBD:U1 snRNP ternary complex. Both the RNAs bind SRSF1-RBD with near equal efficiency due to the presence GGN and $\mathrm{CN}$ ( $\mathrm{N}=$ any nucleotides) motifs (23). We compared the stability of the ternary complexes with that of the $\beta$-globin:U1 snRNP binary complex by a challenge with the 14 -nt long $\beta$-globin 5'SS RNA. The U1 snRNP-dependent complexes were stable in the presence of the short RNA (Figure 1E - compare lanes 3 and 6). However, the relative ratio of the two shifted bands 
changed suggesting that these bands represent conformational variants that react to the 5'SS challenge differently. We observed similar phenomenon when the ternary complex was challenged with Ron ESE (Supplementary Figure S1D). As we noted before with short AdML 5'SS RNA (8), U1 snRNP did not bind strongly to short $\beta$-globin 5'SS RNA after displacing SRSF1-RBD (Supplementary Figure S1E). In contrast to the ternary complex, we found that the U1 snRNP: $\beta$-globin binary complexes were partially disrupted upon addition of excess 14-nt long $\beta$-globin 5'SS RNA (Figure 1F - compare lanes 1, 2, 3 with 4, 5, 6, respectively).

Overall, these results suggest that U1 snRNP recognizes a global 3D structural scaffold of $\beta$-globin, which is not severely impacted by the mutation in the authentic and cryptic $5^{\prime} S S$, and that SRSF1 enhances the global interaction of U1 snRNP to $\beta$-globin (Figure $1 \mathrm{G}$ ).

\section{Cooperative multicopy binding of SRSF1-RBD to $\beta$-globin is important for stabilization of}

\section{U1 snRNP}

To better understand how SRSF1-RBD stabilizes U1 snRNP, we tested concentrationdependent binding of SRSF1-RBD to $10 \mathrm{pM} \beta$-globin probe by EMSA. An apparent-binding saturation and an apparent super-saturation were observed at $\sim 40 \mathrm{nM}$ and $\sim 70 \mathrm{nM}$ protein concentrations, respectively (Figure 2A). In contrast to this high-affinity binding, $K_{d}$ of the SRSF1-RBD:ESE complex is around $0.5 \mu \mathrm{M}$ (24). Consistently, we also found that even at 240 nM, SRSF1-RBD could not saturate 14-nt long Ron ESE (Figure 2B), a strong binding site for SRSF1 (24). This suggests a strong cooperation among SRSF1-RBD molecules for binding to $\beta$-globin. Under stoichiometric binding conditions, with $100 \mathrm{nM} \beta$-globin $\left(>\mathrm{K}_{\mathrm{d}}\right)$, the $\beta$-globin complex represented in the most compact band on the native gel in Supplementary Figure S2A have about 28 molecules of SRSF1-RBD bound to the RNA in consistence with a previous report (6). Similar result was also obtained with $A d M L$ pre-mRNA (Supplementary Figure S2B), which binds about 10 molecules of SRSF1-RBD for an apparent saturation (6). 
Then we examined if a lower level of SRSF1-RBD could form the stabilized ternary complex. Titration of $25 \mathrm{nM} \beta$-globin probe bound to 22X SRSF1-RBD with U1 snRNP indicated that about 60X U1 snRNP was required for generating the well-resolved ternary complexes (Supplementary Figure S2C). We showed later that excess U1 snRNP displaces majority of the SRSF1-RBD molecules from the pre-mRNA in a stoichiometry-dependent manner resolving the ternary complex on gel (Figure 3 and Supplementary Figure S3). Nonetheless, we next added $60 X$ U1 snRNP to $25 \mathrm{nM} \beta$-globin probe bound to $2 \mathrm{X}, 6 \mathrm{X}, 10 \mathrm{X}, 14 \mathrm{X}, 18 \mathrm{X}$, or 22X SRSF1-RBD molecules. At low SRSF1-RBD: $\beta$-globin ratio, smeary (i.e., kinetically unstable) complexes were formed, which migrated between the U1 snRNP-dependent ternary and the U1 snRNPdependent binary complexes (Figure 2C, compares 7 and 13). It appears that roughly 10X SRSF1-RBD is required to form a stable ternary complex formation with U1 snRNP (Figure 2C, compare lanes 9 and 11). Thus, a certain SRSF1: $\beta$-globin ratio is required for SRSF1-RBD to stabilize U1 snRNP and 'lock' itself in a stable complex.

Taken together, these results suggest that multiple SRSF1 molecules could bind the premRNAs in a cooperative mode and a minimum number of SRSF1 molecules are required for stabilization of U1 snRNP (Figure 2D).

\section{Multiple U1 snRNP particles displace majority of SRSF1-RBD molecules and specify the splicing substrate for U2AF65}

To further characterize the process of the ternary complex formation, we titrated $10 \mathrm{pM}$ $\beta$-globin bound to about 25 molecules of SRSF1-RBD with U1 snRNP. Increasing concentrations of U1 snRNP gradually increased the mobility of the pre-mRNA complex, eventually forming the faster migrating complexes (marked with arrows in Figure 3A). Addition of anti-SRSF1 antibody to these complexes exhibited an exponential increase in the migration rate of the super-shifted complexes with increasing concentrations of U1 snRNP up to $80 \mathrm{nM}$ (lanes 3, 5, and 7 of Figure 3B). This suggests that SRSF1-RBD molecules are depleted from $\beta$ - 
globin pre-mRNA with increasing concentrations of U1 snRNP. We also observed that U1 snRNP displaces SRSF1-RBD from short SRSF1-binding RNA such as 14-nt long $\beta$-globin 5'SS RNA (Supplementary Figure S1D) and 14-nt long Ron ESE (Supplementary Figure S3A). To more clearly demonstrate whether U1 snRNP displaces SRSF1-RBD from the full-length premRNA, we wanted to identify a U1 snRNP variant that can displace SRSF1-RBD from the premRNA without itself getting recruited, which would release the free pre-mRNA that can be easily detected by EMSA. Since different protein components of U1 snRNP play important roles in U1 snRNP binding to the RNA (22), we assembled U1 snRNP with varied components to identify a variant that would not bind the pre-mRNA. We found that assembling U1 snRNP with Sm $B_{1-174}$ and without U1-A (U1 snRNP $B_{174} \triangle A$ ) served this purpose. We then titrated SRSF1-RBDsaturated $\beta$-globin with U1 snRNP $B_{174}$ and U1 snRNP $B_{174} \triangle A$ (Figure $3 C$ ). While U1 snRNP $\mathrm{B}_{174}$ formed complexes of similar migration pattern as observed with U1 snRNP (lanes $5,6,7$ ), U1 snRNP $B_{174} \triangle \mathrm{A}$ at high concentrations displaced all SRSF1-RBD molecules from $\beta$-globin releasing free probe (lane 12).

Finally, we examined how titration with U1 snRNP impacts specific binding of U2AF65 that recognizes the PPT and dictates recognition of the 3'SS. Therefore, we added U2AF65 to the ternary complex of $\beta$-globin formed with 160 nM U1 snRNP (Figure 3D - lanes 4-6). Addition of U2AF65 upshifted all U1 snRNP-dependent bands to a single band (lane 4). Titration of the quaternary complex with increasing concentrations of $U 1$ snRNP slightly downshifted the complex likely due to displacement of additional SRSF1-RBD molecules. Interestingly, with $\beta$ globin $\triangle \mathrm{PPT}$, we observed that with increasing concentrations of U1 snRNP, the quaternary complex becomes weaker likely at the expense of large aggregate-like dead-end/non-productive complexes that did not enter the gel $(19,25)$ (Figure 3D - lanes 10-12). We did not observe any detectable binding of 25 nM U2AF65 to protein-free $\beta$-globin pre-mRNA (Supplementary Figure S3B) 
Overall, these results suggest that although a large number of SRSF1-RBD molecules could bind each $\beta$-globin molecule, only a sub-set of these molecules are essential for U1 snRNP recruitment and even a fewer are 'locked' in the early spliceosomal complexes (Figure $3 E)$. We further found that additional copies of U1 snRNP could release the loosely bound SRSF1-RBD molecules and enhances specificity for U2AF65.

\section{Different SR proteins regulate the efficiency of U1 snRNP recruitment to $\beta$-globin through collaboration}

We wished to understand if SRSF1, SRSF2, and SRSF5, all of which promote the splicing $\beta$-globin (21), could cooperate with each other to stably recruit U1 snRNP. For the assembly experiments, we used the RNA binding domains (RBD) of SRSF1 and SRSF5, which are known to be their functional variants $(8,26)$, and SRSF2-RBD in chimera with the fully phosphomimetic RS domain of SRSF1 (SRSF2-RE) (27) (Supplementary Figure S4A). SRSF2RE cooperatively bound $\beta$-globin at $<3 \mathrm{nM}$ concentration (Supplementary Figure S4B, lanes 1-9) while its affinity for its cognate ESE is weaker $\left(\mathrm{K}_{d}=\sim 0.3 \mu \mathrm{M}\right)(28)$. The complex forming the most compact band on the native gel has about 10 molecules of SRSF2-RE bound to it (6). Similarly, at least a few copies of SRSF5-RBD bound to $10 \mathrm{pM} \beta$-globin below $6 \mathrm{nM}$ concentration (Supplementary Figure S4B, lane 11). Binding of SRSF5-RBD to $25 \mathrm{nM}$ unlabeled $\beta$-globin traced with radiolabeled $\beta$-globin indicates that the most compact band is formed with about 15X SRSF5-RBD (Supplementary Figure S4B, lane 23). For all three SR proteins, at high SR protein to pre-mRNA probe ratios, a portion of the probe was caught in the well likely due to formation larger complexes that could not enter the gel.

Then we tested if a suboptimal SRSF1-RBD:pre-mRNA ratio, which fails to stably recruit U1 snRNP, could be compensated for by other SR proteins. For this, we compared the U1 snRNP-dependent $\beta$-globin complexes formed with different stoichiometry of SRSF1-RBD and 
SRSF2-RE. It was evident that even 2-3X molar excess of SRSF1-RBD could stably recruit U1 snRNP if at least $7 X$ SRSF2-RE was present (Figure 4A, lanes 8-13). To further test if the presence of a third SR protein helps stabilize the ternary complex at a different ratio, we added SRSF5-RBD to the binding reaction mixture. In the presence of SRSF5-RBD, SRSF2-RE could be lowered to obtain similar stable ternary complex (Supplementary Figure S4C). We then examined if similar phenomenon could be recapitulated with low concentrations of components in accord with the in vivo milieu. SRSF2-RE and SRSF5-RBD compensated for low concentrations of SRSF1-RBD for formation of the stable U1 snRNP-dependent complexes (Figure 4B). Finally, we observed that the $\beta$-globin complexes formed with U1 snRNP and different SR proteins were not disintegrated by $80,000 X$ molar excess of free $\beta$-globin 5 'SS RNA suggesting its stability (Supplementary Figure S4D).

We also tested if SRSF2-RE or SRSF5-RBD alone could stabilize U1 snRNP on $\beta$-globin. Titration of $\beta$-globin saturated with either SRSF2-RE or SRSF5-RBD with U1 snRNP gradually downshifted the $\beta$-globin complexes forming smears (Supplementary Figure S4E). A smear indicates kinetically unstable complexes unlike as observed with SRSF1-RBD. Thus, it appears that U1 snRNP displaces both SRSF2-RE and SRSF5-RBD, but they alone cannot stabilize U1 snRNP. We also repeated the same experiment with U2AF65 as in Figure 3D but instead of SRSF1-RBD, we used SRSF2-RE (Supplementary Figure S4F). The complexes formed with the $\beta$-globin variants did not enter the gel efficiently. Given the pore size of a $4 \% \mathrm{gel}$ is $>100 \mathrm{~nm}$ (29), we conclude that SRSF2-RE promoted the formation of predominantly the aggregate-like complexes even with WT $\beta$-globin. Then we examined the U2AF65-dependent complex formation with a combination of $10 \mathrm{nM}$ SRSF2-RE and $20 \mathrm{nM}$ SRSF1-RBD (Figure 4C). The non-aggregated complex formed most with WT substrate (lane 2) and to a lesser extent with the $\triangle \mathrm{BS}$ and $\Delta 3^{\prime} \mathrm{SS}$ mutant (lanes 6 and 10 ). This complex was more weakened with $\Delta \mathrm{PPT}$ and $\Delta 5$ 'SS. This observation correlates well with in vivo splicing efficiency of the $\beta$-globin mutants 
(Figure $4 \mathrm{D}$ ). The $\Delta 5$ 'SS and $\triangle \mathrm{PPT}$ mutants were completely defective in splicing while $\Delta \mathrm{BS}$ spliced weakly into WT mRNA likely using a cryptic BS (30) while $\Delta 3^{\prime} S S$ mutant spliced weakly using a cryptic 3'SS 26-nt downstream of the authentic 3'SS (determined by Sanger sequencing).

We then examined if the used SR proteins could form pre-mRNA-independent complexes with U1 snRNP to examine if, upon displacement, they could sequester U1 snRNP into an SR:U1 complex. We previously observed detectable interactions of U1 snRNP with SRSF1-RBD but not SRSF5-RBD in a pre-mRNA-independent manner (8). Further GST pulldown assay indicates that U1 snRNP also forms pre-mRNA-independent complexes with SRSF2-RBD (Figure 4E).

Overall, these results suggest that the stoichiometry of individual SR proteins dictates U1 snRNP stabilization efficiency and U1 snRNP level required for SR protein displacement (Figure 4F).

\section{SR proteins collectively remodel the 3D structural scaffold of the pre-mRNA}

To examine if the differential impacts of varying stoichiometries of SR proteins are mediated by pre-mRNA structural remodeling, we carried out SHAPE analyses of $\beta$-globin bound to 15X SRSF1-RBD (15S1), 4X SRSF1-RBD (4S1), 4X SRSF1-RBD + 12X SRSF2-RE (S1S2), 4X SRSF1-RBD + 8X SRSF5-RBD (S1S5), 4X SRSF1-RBD + 6X SRSF2-RE + 4X SRSF5-RBD (S1S2S5), 15X SRSF2-RE (15S2), and 15X SRSF5-RBD (15S5). The 4S1, 15S2, and 15S5 provide suboptimal conditions for U1 snRNP stabilization while 15S1, S1S2, S1S5, and S1S2S5 are optimal for the same (Figure 4, Supplementary Figure S4).

We calculated fold-changes in the SHAPE reactivity of $\beta$-globin under all experimental conditions compared to $\beta$-globin+SR protein buffer. Figure $5 \mathrm{~A}$ indicates the fold changes in SHAPE reactivity in the optimal samples (15S1, S1S2, S1S5, and S1S2S5). We then identified 
5-nt long segments across the pre-mRNA within which at least one nucleotide exhibits $>1000$ fold enhancement of SHAPE reactivity and the central nucleotides of these segments were given a score of 1 (Supplementary Figure S5A). Where at least 1000-fold enhancement of SHAPE reactivity was not observed upon SR protein binding within two-nucleotides flanking the central nucleotide, it was given a score of zero. The four optimal samples exhibited a score of 1 at the same 105 sites (identified with blue circles in the plot in Figure 5B). We then compared the fold-changes in SHAPE reactivity in the sub-optimal samples and any of these 105 sites that did not exhibit the same property as the optimal samples were given a score of negative 1 and any site that did exhibit a similar property as the optimal samples were given a score of positive 1. All other sites were given a score of zero in the sub-optimal samples. 33 sites in Sample $4 \mathrm{~S} 1$ (Figure 5B), 20 in $15 \mathrm{~S} 5$ (Figure 5C), and 11 in 15S2 (Figure 5D) scored negative 1.

Since at least a small level of SRSF1-RBD was required for a stable binding of U1 snRNP to $\beta$-globin under all tested SR protein combinations, we asked if SRSF1-RBD exhibits any common difference from both SRSF2-RE and SRSF5-RBD. We found that binding of multicopy SRSF1-RBD to $\beta$-globin intron is more cooperative and efficient than that of SRSF2RE as well as SRSF5-RBD (Supplementary Figure S5B, S5C). This suggests that SRSF1-RBD remodels the $3 \mathrm{D}$ structural scaffold of $\beta$-globin differently from the two other SR proteins.

In all, these results suggest that different combinations of SR proteins could collectively remodel the pre-mRNA to generate a pre-mRNA 3D scaffold with common structural features.

\section{RNP reactivity verifies SR protein-mediated pre-mRNA structural remodeling for the stable recruitment of U1 snRNP}

We examined the secondary structural features of $\beta$-globin pre-mRNA in vivo prior to binding of U1 snRNP. To accomplish this, DMS reactivity (DMS modifies single-stranded A and C residues not bound to proteins) was measured after blocking U1 snRNP binding using an antisense morpholino oligonucleotide complementary to the $5^{\prime}$ end of U1 snRNA (8) to observe 
the effect of binding of splicing regulatory RNA binding proteins. The analysis of the DMS-MaPseq data exhibited a high signal-to-noise ratio with zero reactivity for all nucleotides in the untreated sample. Upon DMS-treatment, out of $179 \mathrm{~A}$ and $\mathrm{C}$ residues, only five nucleotides had a zero reactivity and only 35 were modified in less than $2 \%$ of the RNA molecules; the remaining nucleotides were more heavily modified (Supplementary Figure S6A). Given that an open base has a probability of $2-10 \%$ of being modified by DMS (31), this reactivity profile of $\beta$ globin suggests that majority of the nucleotides are open in vivo prior to U1 snRNP recruitment.

Then we wanted to investigate the sites of stoichiometric binding of specific SR proteins and its effects on pre-mRNA structure and U1 snRNP recruitment in vitro. Purification of the premRNA:SR:U1 snRNP complexes was required for its SHAPE and RNP-MaP analysis (see Methods), which could not be done efficiently for $\beta$-globin. Therefore, we tested the mechanisms with $A d M L$ by SHAPE and RNP-MaP, whose complexes could be efficiently purified as described before (8). U1 snRNP binding to AdML apparently saturated with SRSF1-RBD also involves displacement of SRSF1-RBD as was evident from an EMSA result we showed earlier (8). We compared fold changes in SHAPE reactivity of AdML upon binding of 5X SRSF1-RE (SHAPE_S1, the optimal SRSF1 stoichiometry (8)), 2X SRSF1-RE (SHAPE_S1_low suboptimal SRSF1 stoichiometry), U1 snRNP (SHAPE_U1), and 5X SRSF1-RE + U1 snRNP (SHAPE_S1+U1). Fold-changes in SHAPE reactivity in SHAPE_S1 and SHAPE_S1_low compared to protein-free $A d M L$ were different (Figure 6A). SHAPE_S1 exhibits more efficient enhancement of nucleotide flexibility across the pre-mRNA than SHAPE_S1_low, particularly immediately upstream of the 5'SS (marked with a rectangle above the plot). Nucleotide flexibility of this segment was lowered in SHAPE_S1+U1 (Figure 6B). This suggests that to promote the likely contact between U1 snRNP and this segment, SRSF1-mediated remodeling enhances flexibility of the segment immediately upstream of the $5^{\prime} S S$, a segment whose singlestrandedness is important for recruitment of SR proteins (6) and U1 snRNP [(8), this work]. 
Finally, we compared the fold changes in SHAPE reactivity in SHAPE_U1 and SHAPE_S1+U1 (Figure 6C). SHAPE_S1+U1 exhibited a much higher level of flexibility-gain across the premRNA compared to SHAPE_U1. These current observations is consistent with our previous report which conform to the fact that splicing competent pre-mRNAs are highly flexible in vivo (6) (Supplementary Figure S6A) correlating the SR protein-mediated pre-mRNA structural remodeling observed in vitro with the in vivo structural state of the pre-mRNA.

We then examined the protein contacts with the RNA by RNP-MaP-seq, which identifies the nucleotides within $\sim 12 \AA$ of the central carbon atom of an interacting lysine residue, under three different binding conditions: 5XSRSF1 alone (RNP_S1), U1 snRNP alone (RNP_U1), and 5X SRSF1 and U1 snRNP (RNP_S1+U1) (Figure 6D). RNP_S1 exhibits lysine contacts at 44 sites, RNP_S1+U1 at 40 sites, and RNP_U1 at 23 sites. While RNP_U1 exhibits RNP reactivity across the exon 1 and intron, the reactivity is extended into the entire length of the pre-mRNA including exon 2 in RNP_S1 and RNP_S1+U1. Although in RNP_S1+U1, it is not possible to discern if the cross-linking to a nucleotide is mediated by SRSF1 or a protein subunit of U1 snRNP, nucleotides at positions $38,55,85,94,106,108,122,133,149,159,179,180,181$, and 189 exhibit RNP-reactivity only in RNP_S1+U1. The unique contacts in RNP_S1+U1 could be mediated by U1 snRNP components in the presence of SRSF1. This, in turn, suggests that SRSF1-indcued structural remodeling enhances U1 snRNP binding. Additionally, RNP_S1 exhibited RNP reactivity at 43 positions, 24 of which did not show a reactivity in RNP_S1+U1, which could be due to displacement of SRSF1 from the pre-mRNA upon addition of U1 snRNP. Moreover, 17 sites $(25,31,41,43,60,61,62,83,87,124,148,151,170,199,202,205,216)$ exhibit RNP reactivity in both RNP_S1 and RNP_S1+U1 but the reactivity is higher in RNP_S1+U1 suggesting a higher stability of the latter. Similarly, 9 sites $(40,41,54,59,62,93$, 97, 124, 139) exhibit higher RNP reactivity in RNP_S1+U1 than RNP_U1. Three sites - 41, 62, 124 - exhibit RNP reactivity in all three samples. This suggests that SRSF1-mediated U1 
snRNP stabilization to $A d M L$ is a global phenomenon involving cooperation as well as competition between SRSF1 and U1 snRNP at different sites. The protein contacts are also mapped onto the SHAPE-derived secondary structure model of AdML pre-mRNA (Supplementary Figure S6B, C, D).

Finally, we compared the RNP-reactivity in RNP_S1+U1 and the fold-changes in SHAPE reactivity in SHAPE_S1 (Figure 6E). At least 1000-fold enhancement of SHAPE reactivity was observed in SHAPE_S1 within two-nucleotides flanking 12 sites that exhibited RNP reactivity in RNP_S1+U1 but not in RNP_S1 $(40,54,55,59,85,97,122,133,139,179,180,189)$. Among these 12 sites, 11 sites exhibit stable lysine contact in RNP_S1+U1 (except for 97) i.e., they do not exhibit $>1000$-fold increase in SHAPE reactivity in SHAPE_S1+U1. These results corroborate the fact that remodeling of $A d M L$ by SRSF1 enhances the flexibility of various nucleotides across the pre-mRNA for enhanced contacts with U1 snRNP.

Overall, SHAPE- and RNP-reactivity together provide a clearer mechanistic insight into the recruitment of $U 1$ snRNP to $A d M L$ by remodeling of the pre-mRNA 3D structural scaffold by SRSF1. 


\section{DISCUSSION}

How the splicing machinery distinguishes between the highly degenerate splice signal sequences from background sequences have remained a mystery for decades. In the current study we examined the early steps of U1 snRNP recruitment to $\beta$-globin pre-mRNA by EMSA, SHAPE, RNP-MaP, and pull-down assay in the presence of different SR proteins which promote splicing of the test substrates. Our study provides the following insights regarding the regulation of splicing substrate definition by SR proteins.

Multicopy cooperative binding of SR proteins: A minimum number of copies of different premRNA-specific SR protein molecules must bind both the exonic and the intronic motifs in appropriate combinations for an effective pre-mRNA structural remodeling. This is in contrast to the assembled early spliceosome where only one or two ESEs are occupied by a specific SR protein (19). Multiple molecules of individual SR proteins bind the pre-mRNA cooperatively, which compensates for the weak affinity of SR proteins for their cognate binding sites (about 0.5 $\mu \mathrm{M}$ for SRSF1 (24) and $0.3 \mu \mathrm{M}$ for SRSF2 (28)). The cooperative binding occurs likely through a dynamic RNA-protein-protein-RNA interactions and/or cycles of binding and structural remodeling for binding to occluded/weaker sites. We previously observed that recruitment and assembly of these SR:pre-mRNA complexes is often regulated by single-stranded segments immediately upstream of the 5 'SS, which regulate splicing across the transcriptome (6). Our current work suggests this segment could be critical for SR protein-mediated U1 snRNP recruitment. Nonetheless, the number of SR protein molecules that could bind each pre-mRNA molecule appeared to be much higher than what could be predicted by counting the occurrence of in vitro selected binding sites or identified ESEs due to cooperative nature of the binding. This does not mean that the SR protein binding is completely non-specific but rather that the repertoire of known strong and weak binding sites is incomplete (32). 
The diversity of pre-mRNA 3D structural scaffolds and its remodeling by SR proteins: With a human adenovirus 2 pre-mRNA, we recently demonstrated that the 3D structural scaffold of a pre-mRNA could be an integral component of the mammalian splicing code beyond the splice signal sequences, splicing regulatory sequences, and secondary structure regulating the exposure/occlusion/proximity of the last two (8). We also showed that the functionality of this 3D structural scaffold in recruiting early spliceosomal components could be modulated by individual SR proteins. In the current work, we reaffirmed the role of the 3D structural scaffold with a mammalian splicing substrate human HBA IVS I, provided a glimpse of its diversity, and mechanistically correlated it with the widespread functionalities of the SR proteins (33) and the single-stranded segments immediately upstream of the 5'SS (6).

We observed that U1 snRNP could recognize the remodeled 3D structural scaffold of $\beta$ globin comprised of the $5^{\prime}$ exon and the intron. During co-transcriptional recruitment of U1 snRNP, it is reported to remain bound to both the $5^{\prime}$ SS and the elongating RNA polymerase II until after the transcription of the 3' exon starts (34). Thus, recognition of the remodeled splicingconducive structural scaffold of the $5^{\prime}$ exon and the intron by U1 snRNP could be a plausible widespread mechanism in vivo.

Mutation in both $5^{\prime}$ and $3^{\prime}$ splice signals do not strongly inhibit U1 snRNP binding to fulllength $\beta$-globin. This suggests that $\beta$-globin has a loose global 3D structure that is not easily disrupted by splice signal mutations. Additionally, since binding of U1 snRNP to protein-free WT and $\Delta 5$ 'SS $\beta$-globin increases the structural similarity of the two variants within the intron and the $3^{\prime}$ exon, we surmise that $\beta$-globin is assembled into a compact $3 D$ structural scaffold upon binding of U1 snRNP. In contrast, AdML pre-mRNA fails to recruit U1 snRNP upon mutation of any of the four major splice signals (8). This suggests that the 3D structural scaffold of $A d M L$ is compact and strong and is easily disrupted by mutation in any of the splice signals. This also 
suggests that a mutation in the splice signal in pre-mRNAs like $A d M L$ would have inhibited spliceosome assembly at an earlier stage compared to pre-mRNAs like $\beta$-globin.

The question remains if the concept of a remodeled pre-mRNA 3D structural scaffold for recruitment of early spliceosomal components could be valid for introns that are several kilobases long. A recent report suggests that the kinetics of removal of the same intron in the same cells varies greatly and the introns could be progressively removed by recursive splicing across the transcriptome (35). The selection of the relatively short recursive introns could be mediated by recognizable remodeled 3D structural scaffolds. Additionally, the variable rate of intron removal could be due to RNA structure-mediated RNA-protein interaction dynamics $(5,36)$. SR protein displacement by U1 snRNP: The recruited U1 snRNP contacts nucleotides across the pre-mRNA, potentially substituting the SR protein molecules at certain positions. The remaining bound SR protein molecules are displaced from the pre-mRNA by additional stoichiometric level of U1 snRNP particles. The displacement likely occurs due to the ability of U1 snRNP components to weakly interact with various segments of the pre-mRNA. These RNA:U1 snRNP interactions Individually are not sufficiently strong to promote binding of the participating U1 snRNP particles to the pre-mRNA. However, these interactions of an excess of U1 snRNP particles could displace weakly bound SR proteins from across the pre-mRNA. Since U1 snRNP is present in vast excess as compared to other U snRNPs in vivo (37) and in vitro RNA-protein interactions are faithful representations of their in vivo functionalities (38), this is likely an essential novel function of U1 snRNP for spliceosome assembly. Thus, U1 snRNP and SR proteins exhibit cooperative as well as competitive relationships for global stable engagement of U1 snRNP.

U2AF65 binding to splicing-defective splice signal mutants of $\beta$-globin led to formation of large aggregates in a U1 snRNP stoichiometry-dependent manner. We also observed that U1 snRNP binding to protein-free WT and $\Delta 5$ 'SS mutant of $\beta$-globin involved very similar global 
contact except for segments around the 5'SS. Based on these results, we hypothesize that U1 snRNP binding to a pre-mRNA remodeled by SR proteins and subsequent SR protein displacement (as opposed to U1 snRNP binding to a protein-free pre-mRNA) leads to a splicingconducive 3D structure only for a bona fide splicing substrate. Given that U1 snRNP amply binds non-coding RNAs (39), this mechanism could be important for impeding wasteful spliceosome assembly on non-coding RNAs.

U1 snRNP:SR protein interactions: This dynamic process greatly enhances the affinity of U1 snRNP for the pre-mRNA. Currently, it is not entirely clear whether an interaction between the pre-mRNA-bound U1 snRNP and the pre-mRNA-bound SR protein is important for U1 snRNP stabilization at this stage. We have observed that SRSF1 that can strongly interact with U1 snRNP in a pre-mRNA-independent manner alone could stabilize U1 snRNP on WT $\beta$-globin (this work) and WT AdML (8). However, SRSF5-RBD but not SRSF1 stabilizes U1 snRNP on a 3'SS mutant of AdML that splices using a cryptic 3'SS 6-nt downstream of the authentic 3'SS with WT-like efficiency (8). Interestingly, SRSF5-RBD does not exhibit a detectable pre-mRNAindependent interaction with U1 snRNP. SRSF2-RBD, which can interact with U1 snRNP cannot stabilize $\beta$-globin:U1 snRNP interactions although $\beta$-globin contains SRSF2-specific ESEs and is responsive to SRSF2-dependent splicing activation (40). This suggests that the effectiveness of an SR protein in activating splicing is also regulated by the splicing-conducive 3D structural scaffold.

Additionally, the pre-mRNA-independent interactions between certain SR proteins and U1 snRNP would lead to formation of binary complexes upon the displacement of the SR proteins by U1 snRNP. This could disrupt the functions of free U1 snRNP particles in vivo upon elevation of expression of these SR proteins. Further research is required for functional analysis of this phenomenon. 
Potential mechanism of splicing inhibition by SR proteins: Since there is a stoichiometric relationship between the bound SR protein molecules and U1 snRNP particles required to displace them, an increase in SR protein levels without an increase in U1 snRNP level could occlude the pre-mRNA and repress splicing $(41,42)$.

Insights into the mechanisms of splicing regulation by other SRps: Our data explain why the position of individual short degenerate binding motifs of SR proteins individually cannot explain the splicing outcome. It also explains why cooperative binding of these proteins involving homomeric interactions to repeating or 'multivalent' motifs is important. Inability of individual binding motifs to explain the splicing outcome and the cooperative binding are also reported for many other SRps (43-49). Thus, we infer that the mechanistic knowledge presented in the current work could also be extended to a variety of other SRps.

Translational potential of these findings: Expression of the serine-arginine-rich (SR) proteins are tightly regulated in cells and changes in their expression, particularly overexpression, has been shown to transform cells through regulation of alternative splicing of tumor suppressor genes (9-17). The changes in splicing caused by overexpression of individual SR proteins are often explained by concentration-dependent changes in SR protein binding to the RNA motifs. Therefore, currently the therapeutic intervention involves inactivation of the active SR proteins. Since SR proteins are activated upon phosphorylation of the RS domain and consequent exposure the RNA binding domain (RBD) containing the RRMs $(26,50,51)$, most of the inhibitors to counteract SR overexpression in tumors target the kinases that phosphorylate them (52). However, the targets of these kinases include a wide variety of proteins and not limited to one or two specific SR proteins (53). Our work provides evidence for intermolecular interactions of SR proteins (23) to be important for recruitment of early spliceosomal components, highlighting potential targets for SR protein-specific therapeutic intervention. 
Overall, this work provides a greater understanding of concentration-dependent changes in functions of individual SR proteins and potentially other splicing regulatory proteins in splicing activation as well as repression.

\section{AUTHOR CONTRIBUTION}

KS designed and performed the experiments, analyzed the data, and wrote the paper. GG designed some experiments, wrote the paper, and supervised the project.

\section{ACKNOWLEDGEMENT}

The authors would like to acknowledge Dr Simpson Joseph for critical reading of the manuscript and Mike Minh Fernandez for some early work.

\section{FUNDING}

National Institute of General Medical Sciences [R01GM085490]. 


\section{REFERENCES}

1. Will, C.L. and Luhrmann, R. (2011) Spliceosome structure and function. Cold Spring Harb. Perspect. Biol., 3, pii: a003707.

2. Guth, S., Tange, T. .., Kellenberger, E. and Valcárcel, J. (2001) Dual function for U2AF35 in AGdependent pre-mRNA splicing. Mol Cell Biol, 21, 7673-7681.

3. Shao, C., Yang, B., Wu, T., Huang, J., Tang, P., Zhou, Y., Zhou, J., Qiu, J., Jiang, L., Li, H. et al. (2014) Mechanisms for U2AF to define 3' splice sites and regulate alternative splicing in the human genome. Nat Struct Mol Biol, 21, 997-1005.

4. Baralle, M. and Baralle, F.E. (2018) The splicing code. Bio Systems, 164, 39-48.

5. Saldi, T., Riemondy, K., Erickson, B. and Bentley, D.L. (2021) Alternative RNA structures formed during transcription depend on elongation rate and modify RNA processing. Mol Cell, 81, 17891801.e1785.

6. Saha, K., England, W., Fernandez, M.M., Biswas, T., Spitale, R.C. and Ghosh, G. (2020) Structural disruption of exonic stem-loops immediately upstream of the intron regulates mammalian splicing Nucleic Acids Res, 48, 6294-6309.

7. Fu, X.D. and Ares, M., Jr. (2014) Context-dependent control of alternative splicing by RNAbinding proteins. Nat Rev Genet, 15, 689-701.

8. Saha, K., Fernandez, M.M., Biswas, T., Joseph, S. and Ghosh, G. (2021) Discovery of a pre-mRNA structural scaffold as a contributor to the mammalian splicing code. Nucleic Acids Res, 49, 71037121.

9. Kędzierska, H. and Piekiełko-Witkowska, A. (2017) Splicing factors of SR and hnRNP families as regulators of apoptosis in cancer. Cancer letters, 396, 53-65.

10. Sun, S., Zhang, Z., Sinha, R., Karni, R. and Krainer, A.R. (2010) SF2/ASF autoregulation involves multiple layers of post-transcriptional and translational control. Nat Struct Mol Biol, 17, 306-312.

11. Wu, H., Sun, S., Tu, K., Gao, Y., Xie, B., Krainer, A.R. and Zhu, J. (2010) A splicing-independent function of SF2/ASF in microRNA processing. Mol Cell, 38, 67-77.

12. Sokół, E., Kędzierska, H., Czubaty, A., Rybicka, B., Rodzik, K., Tański, Z., Bogusławska, J. and Piekiełko-Witkowska, A. (2018) microRNA-mediated regulation of splicing factors SRSF1, SRSF2 and hnRNP A1 in context of their alternatively spliced 3'UTRs. Experimental cell research, 363, 208-217.

13. Karni, R., de Stanchina, E., Lowe, S.W., Sinha, R., Mu, D. and Krainer, A.R. (2007) The gene encoding the splicing factor SF2/ASF is a proto-oncogene. Nat Struct Mol Biol, 14, 185-193.

14. Das, S. and Krainer, A.R. (2014) Emerging functions of SRSF1, splicing factor and oncoprotein, in RNA metabolism and cancer. Molecular cancer research : MCR, 12, 1195-1204.

15. Luo, C., Cheng, Y., Liu, Y., Chen, L., Liu, L., Wei, N., Xie, Z., Wu, W. and Feng, Y. (2017) SRSF2 Regulates Alternative Splicing to Drive Hepatocellular Carcinoma Development. Cancer research, 77, 1168-1178.

16. Kim, H.R., Lee, G.O., Choi, K.H., Kim, D.K., Ryu, J.S., Hwang, K.E., Na, K.J., Choi, C., Kuh, J.H., Chung, M.J. et al. (2016) SRSF5: a novel marker for small-cell lung cancer and pleural metastatic cancer. Lung cancer (Amsterdam, Netherlands), 99, 57-65.

17. Yang, S., Jia, R. and Bian, Z. (2018) SRSF5 functions as a novel oncogenic splicing factor and is upregulated by oncogene SRSF3 in oral squamous cell carcinoma. Biochimica et biophysica acta. Molecular cell research, 1865, 1161-1172.

18. Zhou, Z. and Fu, X.-D. (2013) Regulation of splicing by SR proteins and SR protein-specific kinases. Chromosoma, 122, 191-207. 
19. Jobbins, A.M., Reichenbach, L.F., Lucas, C.M., Hudson, A.J., Burley, G.A. and Eperon, I.C. (2018) The mechanisms of a mammalian splicing enhancer. Nucleic Acids Res, 46, 2145-2158.

20. Roca, X., Krainer, A.R. and Eperon, I.C. (2013) Pick one, but be quick: 5 ' splice sites and the problems of too many choices. Genes Dev. , 27, 129-144

21. Screaton, G.R., Cáceres, J.F., Mayeda, A., Bell, M.V., Plebanski, M., Jackson, D.G., Bell, J.I. and Krainer, A.R. (1995) Identification and characterization of three members of the human SR family of pre-mRNA splicing factors. EMBO J, 14, 4336-4349.

22. Kondo, Y., Oubridge, C., Roon, A.M.v. and Nagai, K. (2015) Crystal structure of human U1 snRNP, a small nuclear ribonucleoprotein particle, reveals the mechanism of $5^{\prime}$ splice site recognition. Elife, 4, DOI: 10.7554/eLife.04986.

23. Cléry, A., Krepl, M., Nguyen, C.K.X., Moursy, A., Jorjani, H., Katsantoni, M., Okoniewski, M., Mittal, N., Zavolan, M., Sponer, J. et al. (2021) Structure of SRSF1 RRM1 bound to RNA reveals an unexpected bimodal mode of interaction and explains its involvement in SMN1 exon7 splicing. Nat Commun, 12, 428.

24. Cho, S., Hoang, A., Chakrabarti, S., Huynh, N., Huang, D.B. and Ghosh, G. (2011) The SRSF1 linker induces semi-conservative ESE binding by cooperating with the RRMs. Nucleic Acids Res, 39, 9413-9421.

25. Hoskins, A.A., Friedman, L.J., Gallagher, S.S., Crawford, D.J., Anderson, E.G., Wombacher, R., Ramirez, N., Cornish, V.W., Gelles, J. and Moore, M.J. (2011) Ordered and dynamic assembly of single spliceosomes. Science, 331, 1289-1295.

26. Cho, S., Hoang, A., Sinha, R., Zhong, X.-Y., Fu, X.-D., Krainer, A.R. and Ghosh, G. (2011) Interaction between the RNA binding domains of Ser-Arg splicing factor 1 and U1-70K snRNP protein determines early spliceosome assembly. Proc. Natl. Acad. Sci. USA, 108, 8233-8238.

27. Chandler, S.D., Mayeda, A., Yeakley, J.M., Krainer, A.R. and Fu, X.-D. (1997) RNA splicing specificity determined by the coordinated action of RNA recognition motifs in SR proteins. Proc. Natl. Acad. Sci. USA, 94, 3596-3601.

28. Zhang, J., Lieu, Y.K., Ali, A.M., Penson, A., Reggio, K.S., Rabadan, R., Raza, A., Mukherjee, S. and Manley, J.L. (2015) Disease-associated mutation in SRSF2 misregulates splicing by altering RNAbinding affinities. Proc Natl Acad Sci U S A, 112, E4726-4734.

29. Holmes, D.L. and Stellwagen, N.C. (1991) Estimation of polyacrylamide gel pore size from Ferguson plots of normal and anomalously migrating DNA fragments. I. Gels containing $3 \%$ N,N'methylenebisacrylamide. Electrophoresis, 12, 253-263.

30. Pineda, J.M.B. and Bradley, R.K. (2018) Most human introns are recognized via multiple and tissue-specific branchpoints. Genes Dev, 32, 577-591.

31. Tomezsko, P.J., Corbin, V.D.A., Gupta, P., Swaminathan, H., Glasgow, M., Persad, S., Edwards, M.D., Mclntosh, L., Papenfuss, A.T., Emery, A. et al. (2020) Determination of RNA structural diversity and its role in HIV-1 RNA splicing. Nature, 582, 438-442.

32. Anczuków, O., Akerman, M., Cléry, A., Wu, J., Shen, C., Shirole, N.H., Raimer, A., Sun, S., Jensen, M.A., Hua, Y. et al. (2015) SRSF1-Regulated Alternative Splicing in Breast Cancer. Mol Cell, 60, 105-117.

33. Pandit, S., Zhou, Y., Shiue, L., Coutinho-Mansfield, G., Li, H., Qiu, J., Huang, J., Yeo, G.W., Jr, M.A. and Fu, X.-D. (2013) Genome-wide analysis reveals SR protein cooperation and competition in regulated splicing. Mol. Cell, 50, 223-235.

34. Leader, Y., Lev Maor, G., Sorek, M., Shayevitch, R., Hussein, M., Hameiri, O., Tammer, L., Zonszain, J., Keydar, I., Hollander, D. et al. (2021) The upstream 5' splice site remains associated to the transcription machinery during intron synthesis. Nat Commun, 12, 4545. 
35. Wan, Y., Anastasakis, D.G., Rodriguez, J., Palangat, M., Gudla, P., Zaki, G., Tandon, M., Pegoraro, G., Chow, C.C., Hafner, M. et al. (2021) Dynamic imaging of nascent RNA reveals general principles of transcription dynamics and stochastic splice site selection. Cell, 184, 28782895.e2820.

36. Çetin, B., Song, G.J. and O'Leary, S.E. (2020) Heterogeneous Dynamics of Protein-RNA Interactions across Transcriptome-Derived Messenger RNA Populations. Journal of the American Chemical Society, 142, 21249-21253.

37. Burge, C.B., Tuschl, T. and Sharp, P.A. (1999) Splicing of precursors to mRNAs by the spliceosomes. Cold Spring Harbor Monograph Series, 37, 525-560.

38. Taliaferro, J.M., Lambert, N.J., Sudmant, P.H., Dominguez, D., Merkin, J.J., Alexis, M.S., Bazile, C. and Burge, C.B. (2016) RNA Sequence Context Effects Measured In Vitro Predict In Vivo Protein Binding and Regulation. Mol Cell, 64, 294-306.

39. Engreitz, J.M., Sirokman, K., McDonel, P., Shishkin, A.A., Surka, C., Russell, P., Grossman, S.R., Chow, A.Y., Guttman, M. and Lander, E.S. (2014) RNA-RNA interactions enable specific targeting of noncoding RNAs to nascent Pre-mRNAs and chromatin sites. Cell, 159, 188-199.

40. Schaal, T.D. and Maniatis, T. (1999) Multiple distinct splicing enhancers in the protein-coding sequences of a constitutively spliced pre-mRNA. Mol. Cell Biol., 19, 261-273.

41. Busch, A. and Hertel, K.J. (2012) Evolution of SR protein and hnRNP splicing regulatory factors. Wiley Interdiscip Rev RNA, 3.

42. Akerman, M., Fregoso, O.I., Das, S., Ruse, C., Jensen, M.A., Pappin, D.J., Zhang, M.Q. and Krainer, A.R. (2015) Differential connectivity of splicing activators and repressors to the human spliceosome. Genome Biology, 16, 1-18.

43. Ule, J. and Blencowe, B.J. (2019) Alternative Splicing Regulatory Networks: Functions, Mechanisms, and Evolution. Mol Cell, 76, 329-345.

44. Cereda, M., Pozzoli, U., Rot, G., Juvan, P., Schweitzer, A., Clark, T. and Ule, J. (2014) RNAmotifs: prediction of multivalent RNA motifs that control alternative splicing. Genome Biol, 15, R20.

45. Chou, M.Y., Underwood, J.G., Nikolic, J., Luu, M.H. and Black, D.L. (2000) Multisite RNA binding and release of polypyrimidine tract binding protein during the regulation of c-src neural-specific splicing. Mol Cell, 5, 949-957.

46. Ule, J., Jensen, K.B., Ruggiu, M., Mele, A., Ule, A. and Darnell, R.B. (2003) CLIP identifies Novaregulated RNA networks in the brain. Science, 302, 1212-1215.

47. Banani, S.F., Lee, H.O., Hyman, A.A. and Rosen, M.K. (2017) Biomolecular condensates: organizers of cellular biochemistry. Nature reviews. Molecular cell biology, 18, 285-298.

48. Kato, M. and McKnight, S.L. (2018) A Solid-State Conceptualization of Information Transfer from Gene to Message to Protein. Annu Rev Biochem, 87, 351-390.

49. Li, P., Banjade, S., Cheng, H.C., Kim, S., Chen, B., Guo, L., Llaguno, M., Hollingsworth, J.V., King, D.S., Banani, S.F. et al. (2012) Phase transitions in the assembly of multivalent signalling proteins. Nature, 483, 336-340.

50. Tacke, R., Chen, Y. and Manley, J.L. (1997) Sequence-specific RNA binding by an SR protein requires RS domain phosphorylation: creation of an SRp40-specific splicing enhancer. Proc. Natl. Acad. Sci. USA, 94, 1148-1153.

51. Aubol, B.E., Wu, G., Keshwani, M.M., Movassat, M., Fattet, L., Hertel, K.J., Fu, X.D. and Adams, J.A. (2016) Release of SR Proteins from CLK1 by SRPK1: A Symbiotic Kinase System for Phosphorylation Control of Pre-mRNA Splicing. Mol Cell, 63, 218-228.

52. Bates, D.O., Morris, J.C., Oltean, S. and Donaldson, L.F. (2017) Pharmacology of Modulators of Alternative Splicing. Pharmacological reviews, 69, 63-79. 
53. Gou, L.T., Lim, D.H., Ma, W., Aubol, B.E., Hao, Y., Wang, X., Zhao, J., Liang, Z., Shao, C., Zhang, X. et al. (2020) Initiation of Parental Genome Reprogramming in Fertilized Oocyte by Splicing Kinase SRPK1-Catalyzed Protamine Phosphorylation. Cell, 180, 1212-1227.e1214.

54. Mathews, D.H. (2014) RNA secondary structure analysis using RNAstructure. Curr. Protoc. Bioinformatics, 46, 12.16.11-12.16.25.

55. Weidmann, C.A., Mustoe, A.M., Jariwala, P.B., Calabrese, J.M. and Weeks, K.M. (2021) Analysis of RNA-protein networks with RNP-MaP defines functional hubs on RNA. Nat Biotechnol, 39, 347-356.

56. Oh, J.M., Di, C., Venters, C.C., Guo, J., Arai, C., So, B.R., Pinto, A.M., Zhang, Z., Wan, L., Younis, I. et al. (2017) U1 snRNP telescripting regulates a size-function-stratified human genome. Nat Struct Mol Biol, 24, 993-999. 


\section{FIGURE LEGENDS}

Figure 1. SRSF1-RBD stabilizes the interactions between the global 3D structural scaffold of $\beta$-globin and U1 snRNP

(A) $\beta$-globin forms ternary complexes with SRSF1-RBD and U1 snRNP, which migrate primarily as two major bands (marked with black arrows in lane 4); U1 snRNP also binds free $\beta$-globin (lanes 6-8); the ternary complex is super-shifted with aSRSF1 (lane 9, marked with an orange arrow) and aU1-C (lanes 18-19); slightly more smeary complexes of similar migration patterns were formed with $\beta$-globin $\Delta 5$ 'SS (lanes 10-17); red script indicates radiolabeled components; the position of the free probe is indicated with an exon-intron-exon schematic. (B) $\beta g-\Delta E x 2$ ( $\beta$ globin lacking the $3^{\prime}$ exon) formed U1 snRNP-dependent complexes in the presence of $60 \mathrm{nM}$ SRSF1-RBD (the complexes are marked with arrows in lane 3); these U1 snRNP-dependent complexes were significantly weakened for $\beta g-\Delta E x 2$ with 5 'SS mutations ( $\beta g-\Delta E x 2 \Delta 5 ' S S)$ and $\beta g-\Delta E x 2$ with hybridization-mutation immediately upstream of the 5 'SS $(\beta g-\Delta E x 2 \mathrm{EH} 3+4)$ (the position of the weakened complexes are marked with curved brackets in lanes 9 \& 15); U1 snRNP shows no well-defined interaction with the free RNAs (lanes $5,6,11,12,17,18$ ); the position of the free probe is indicated with an exon-intron schematic. (C \& D) Overlaid SHAPE reactivity of protein-free $\beta$-globin WT and its 5 'SS mutant $(\Delta 5)(C)$ and U1 snRNP-bound $\beta$ globin WT and the $\beta$-globin 5'SS-mutant (D); the segments showing a high correlation between moving averages (period $=6$ ) of SHAPE reactivity are marked in each plot and the corresponding $r$ and $p$ values are indicated; exon-intron boundaries are demarcated with dotted vertical lines. (E) The ternary complexes assembled with U1 snRNP mixed with short $\beta$-globin 5'SS RNA ( $\beta g-5$ 'SS) showing formation of compact complexes. (F) Mixing of $\beta$-globin:U1 snRNP binary complexes with short $\beta$-globin 5'SS RNA disintegrates the binary complexes releasing free probe. (G) Take-home message of this figure. 
Figure 2. Cooperative binding of SRSF1-RBD to $\beta$-globin is important for stabilization of U1 snRNP

(A) EMSA showing gradual upshift of radiolabeled $\beta$-globin complexes upon titration with SRSF1-RBD. (B) EMSA showing formation of weak complexes with radiolabeled 14-nt long Ron ESE (a binding site for SRSF1). (C) Varied U1 snRNP stabilization efficiency of 22X, 18X, 14X, 10X, 6X, and 2X SRSF1-RBD bound to $25 \mathrm{nM} \beta$-globin (compare lanes $3,5,7,9,11,13$ ); smeary complexes are kinetically unstable complexes. (D) Take-home message of this figure.

Figure 3. Multiple U1 snRNP particles are required for displacement of majority of SRSF1RBD molecules and specifying the splicing substrate for U2AF65

(A) Titration of SRSF1-RBD-saturated $\beta$-globin with U1 snRNP displaces SRSF1-RBD molecules forming U1 snRNP-dependent complexes (marked with arrows) (lanes 2-13). (B) Super-shift of complexes formed by titrating SRSF1-RBD-saturated $\beta$-globin with U1 snRNP with aSRSF1 showing exponential increase in the migration rate of the super-shifted complexes up to $80 \mathrm{nM}$ U1 snRNP. (C) U1 snRNP assembled with Sm $B_{1-174}$ and without U1-A displaces SRSF1-RBD molecules from $\beta$-globin releasing free pre-mRNA (lanes 2, 8-12). (D) Titration of the quaternary complexes of $\beta$-globin WT and $\beta$-globin $\triangle \mathrm{PPT}$ assembled with SRSF1-RBD, U1 snRNP, and U2AF65 with additional U1 snRNP distinctively changes their migration forming aggregate-like mutant complexes caught in the well; the ratios of the band-intensity of the free pre-mRNA and the quaternary complexes (marked with yellow arrows) are indicated in red script below the image; the mutated sequence in $\triangle \mathrm{PPT}$ is shown. (E) Take-home message of this figure.

Figure 4. SR protein: $\beta$-globin ratio dictates U1 snRNP stabilization efficiency and U1 snRNP level requirement for SR protein displacement

(A) U1 snRNP stabilization efficiency on $25 \mathrm{nM} \beta$-globin with 10X, 8X, 6X, 3X, and 2X SRSF1RBD (lanes 4-7), with 6X, 3X, and 2X SRSF1-RBD and 10X SRSF2-RE (lanes 8-10), with 6X, 
3X, and 2X SRSF1-RBD and 7X SRSF2-RE (lanes 11-13), with 6X, 3X, and 2X SRSF1-RBD and 3X SRSF2-RE (lanes 14-16), with 6X and 3X SRSF1-RBD and 2X SRSF2-RE (lanes 1718). (B) EMSA showing formation of stable U1 snRNP-dependent $\beta$-globin complexes with optimal level of SRSF1-RBD alone (lane 2), with suboptimal level of SRSF1-RBD compensated for by low levels of SRSF2-RE and/or SRSF5-RBD (compare lanes 3 with 4-9). (C) Efficiency of assembly of early spliceosomal complexes with U1 snRNP, U2AF65, SRSF1-RBD, and SRSF2$\mathrm{RE}$ is highest for WT $\beta$-globin, slightly less for splicing-competent splice signal mutants $(\Delta \mathrm{BS}$ and $\Delta 3^{\prime} S S$ ), and least for splicing-defective splice signal mutants ( $\Delta 5$ 'SS and $\left.\Delta P P T\right)$. (D) Transfection-based splicing efficiency of $\beta$-globin WT and splice signal mutants; ‘*' indicates an mRNA produced using a cryptic 3'SS 26-nt downstream of the authentic 3'SS. (E) GST-pull down assay showing pre-mRNA-independent interactions between U1 snRNP and SRSF1-RBD as well as SRSF2-RBD. (F) Take-home message of this figure.

Figure 5. Global structural remodeling of $\beta$-globin with different SR protein combinations (A) Fold changes in SHAPE reactivity upon binding of 15X SRSF1-RBD (15S1), 4X SRSF1RBD + 12X SRSF2-RE (S1S2), 4X SRSF1-RBD + 8X SRSF5-RBD (S1S5), and 4X SRSF1RBD + 6X SRSF2-RE + 4X SRSF5-RBD (S1S2S5) to $\beta$-globin shown for 20-150-nt (top), 150300-nt (middle), 300-420-nt (bottom). (B, C, D) The central nucleotide of 5-nt long segments across the pre-mRNA within which at least one nucleotide exhibits $>1000$-fold enhancement of SHAPE reactivity was given a score of 1 in the optimal samples (15S1, S1S2, S1S5, S1S2S5) (Supplementary Figure S5A); the common 105 nucleotides scored 1 in all optimal samples are indicated with blue circles in B, C, D; in the sub-optimal samples (4S1, 15S2, 15S5), any of these 105 sites that did not exhibit the same property as the optimal samples were given a score of negative 1 and any site that did exhibit a similar property were given a score of positive 1; all other sites were given a score of zero in the sub-optimal samples; 33 sites in sample $4 \mathrm{~S} 1$ 
(orange dotted line in B), 20 in $15 S 5$ (grey dotted line in C), and 11 in $15 S 2$ (yellow dotted line in D) scored negative 1 .

Figure 6. Global structural remodeling and protein contacts involved in U1 snRNP recruitment to $A d M L$

(A) Fold changes in SHAPE reactivity of $A d M L$ upon binding of $5 X$ SRSF1-RE (optimal sample, blue line) or $2 X$ SRSF1-RE (sub-optimal sample, orange line); $\log _{10}$ of fold changes in SHAPE reactivity are plotted along $y$-axis showing both increase (i.e., $y>1$ ) or decrease (i.e., $y<1$ ); the segment immediately upstream of the 5'SS is marked with a rectangle above the plot. (B) Fold changes in SHAPE reactivity upon binding of 5X SRSF1-RE (blue line) or 5X SRSF1-RE + U1 snRNP (yellow line); fold changes in SHAPE reactivity are plotted along $y$-axis showing only the increase (i.e., $y>1$ ). (C) Fold changes in SHAPE reactivity upon binding of U1 snRNP alone (grey line) or 5X SRSF1-RE + U1 snRNP (yellow line). (D) RNP reactivity for AdML bound to U1 snRNP (blue line), SRSF1-RE (orange line), SRSF1-RE + U1 snRNP (grey dotted line); in vitro selected SRSF1-binding sites are demarcated below the plot and exon-intron boundaries are indicated with dotted vertical lines. (E) Fold-changes in SHAPE reactivity of 5XSRSF1$\mathrm{RE}+A d M L$ sample (blue line) plotted along primary $y$-axis and RNP-reactivity of $5 \mathrm{XSRSF} 1+\mathrm{U} 1$ snRNP $+A d M L$ sample (orange line) plotted along secondary $y$-axis.

Figure 7. Proposed model of U1 snRNP and U2AF65 recruitment to the full-length premRNA

Irrespective of whether the global 3D structural scaffold of the pre-mRNA is capable of recruiting U1 snRNP, different pre-mRNA-specific-SRps like SR proteins bind the pre-mRNA cooperatively and structurally remodel the pre-mRNA making it more recognizable by U1 snRNP. Multiple copies of U1 snRNP particles displace the majority of the RBP molecules from the pre-mRNA with concomitant recruitment of U1 snRNP to the remodeled pre-mRNA. 
Interactions of U1 snRNP with the pre-mRNA as well as the RBP are indicated with stars - a solid yellow star indicates top surface and a hollow star bottom surface. U1 snRNP-mediated SRp displacement makes the complex a more suitable substrate for U2AF65. 
bioRxiv Areprint doi: https://doi.org/10.1101/2021.12.01.470860; this version posted December 9, 2021. The copyright holder for this preprint

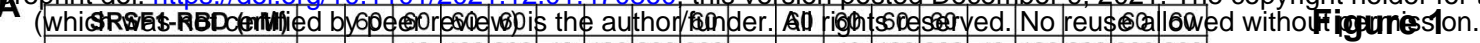

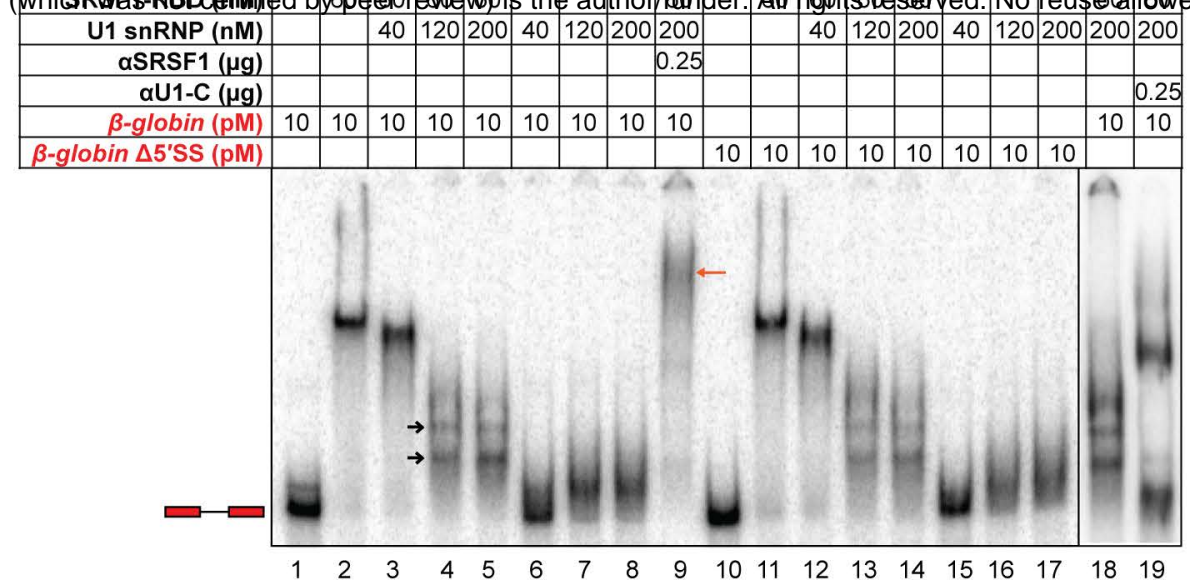

B

\begin{tabular}{|r|c|c|c|c|c|c|c|c|c|c|c|c|c|c|c|c|c|c|}
\hline SRSF1-RBD (nM) & & 60 & 60 & 60 & & & & 60 & 60 & 60 & & & & 60 & 60 & 60 & & \\
\hline U1 snRNP (nM) & & & 80 & 160 & 80 & 160 & & & 80 & 160 & 80 & 160 & & & 80 & 160 & 80 & 160 \\
\hline$\beta g \Delta \mathrm{E} 2(\mathrm{pM})$ & 10 & 10 & 10 & 10 & 10 & 10 & & & & & & & & & & & & \\
\hline$\beta g \Delta \mathrm{Ex} 2 \Delta 5$ 'SS (pM) & & & & & & & 10 & 10 & 10 & 10 & 10 & 10 & & & & & & \\
\hline$\beta g \Delta \mathrm{Ex} 2 \mathrm{EH} 3+4(\mathrm{pM})$ & & & & & & & & & & & & & 10 & 10 & 10 & 10 & 10 & 10 \\
\hline
\end{tabular}

C
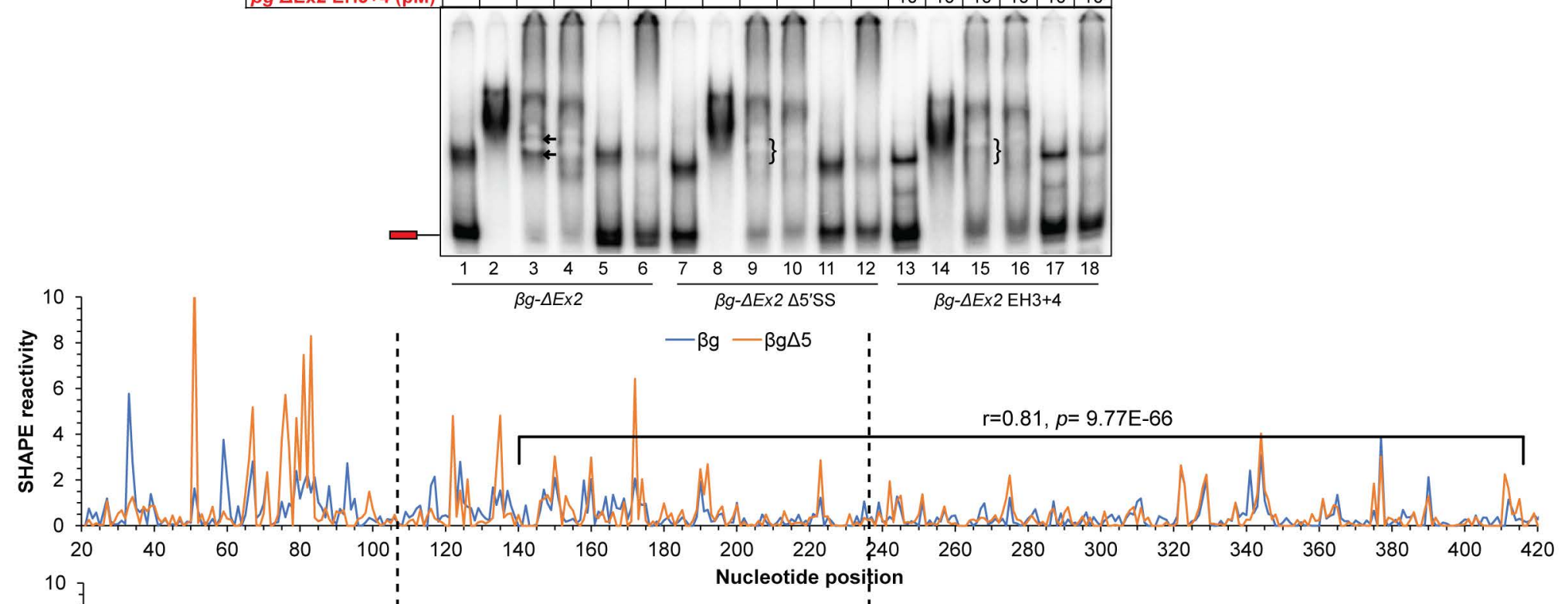

D

$$
\text { s }
$$

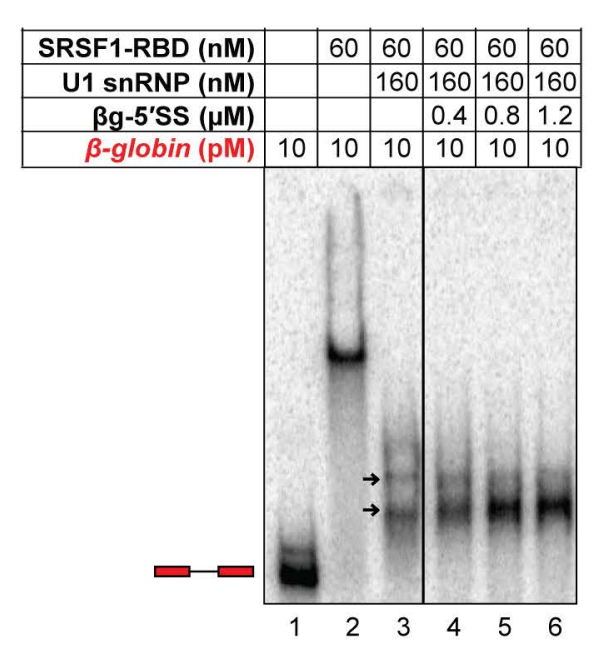


A

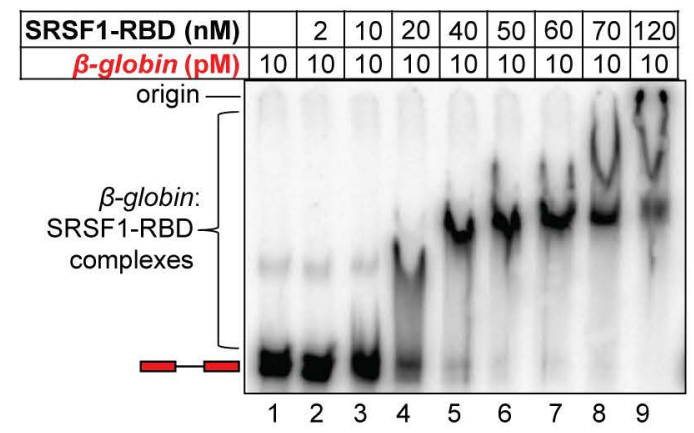

C

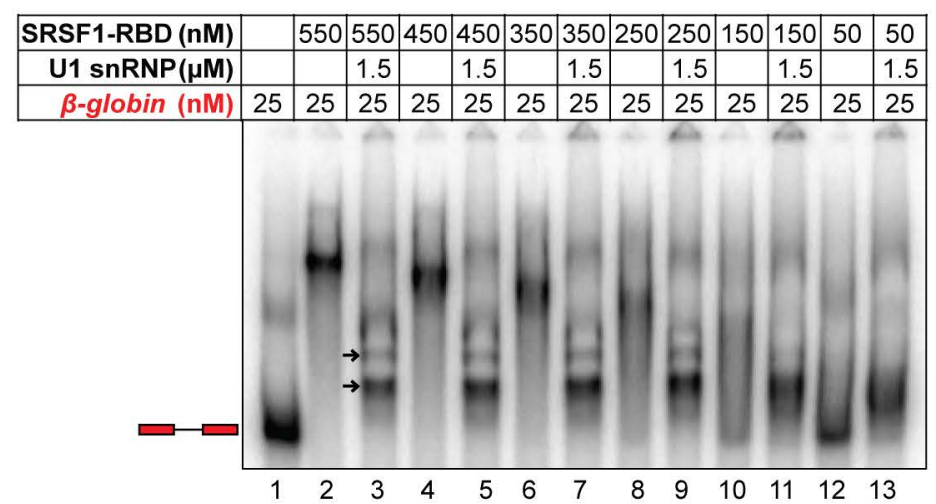

B

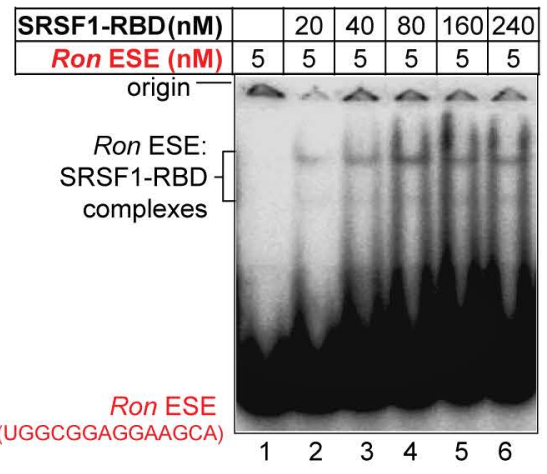

D

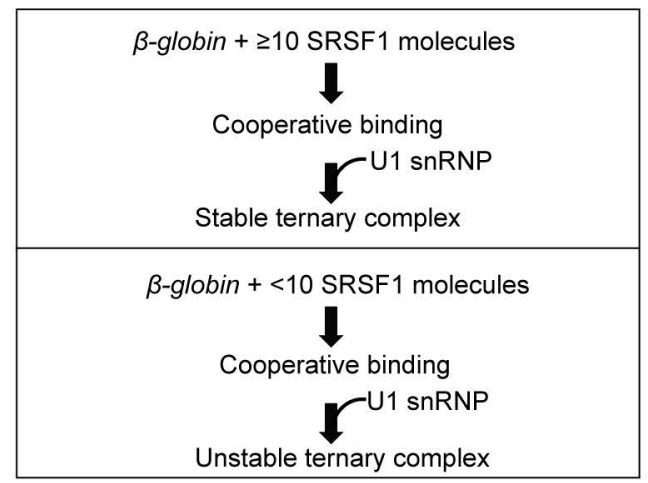


A

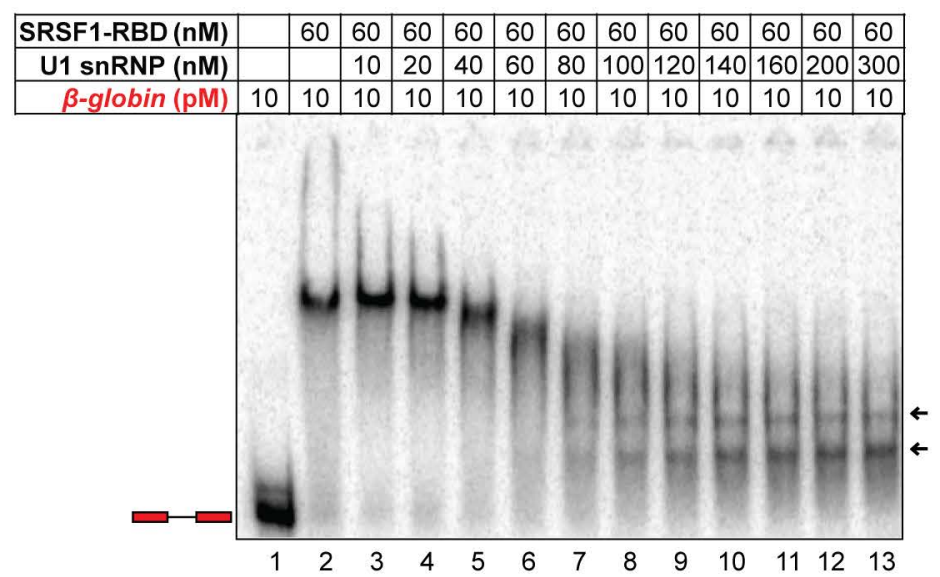

C

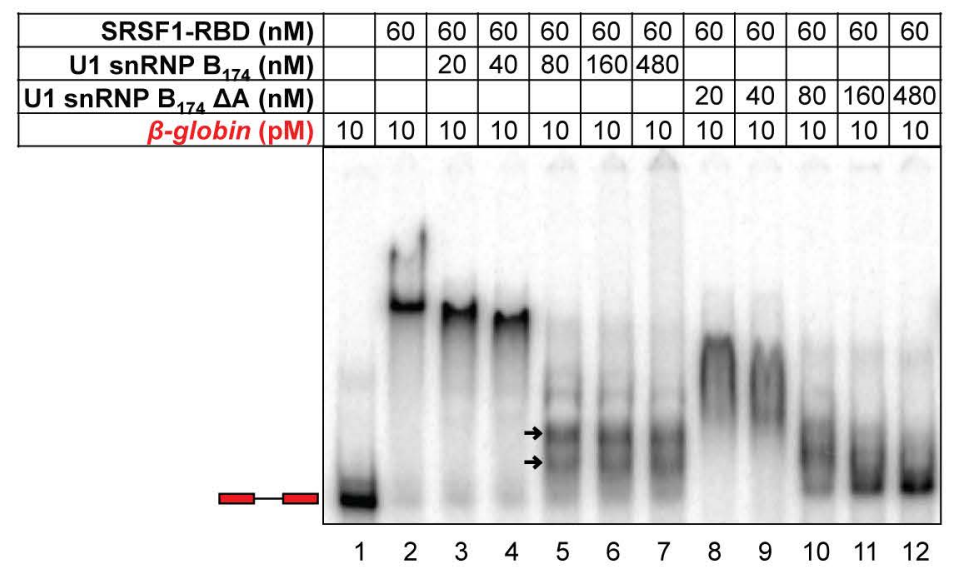

E
B

Figure 3

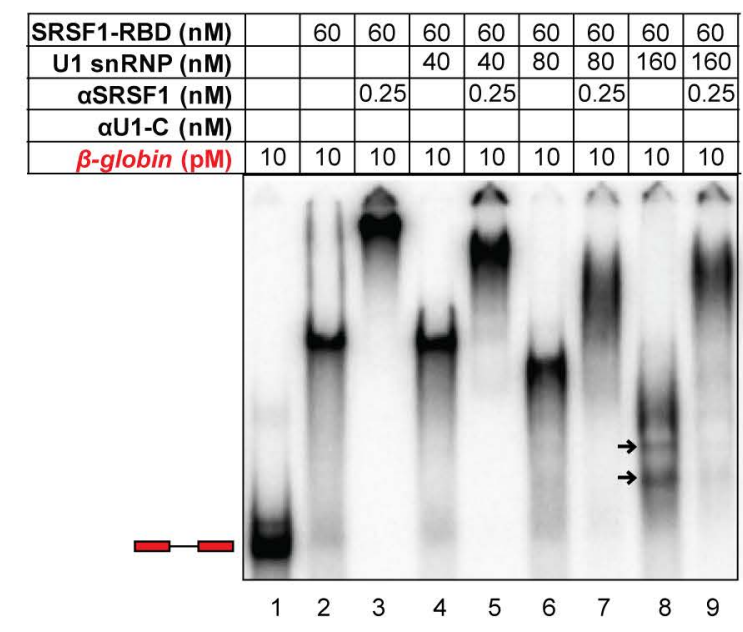

D

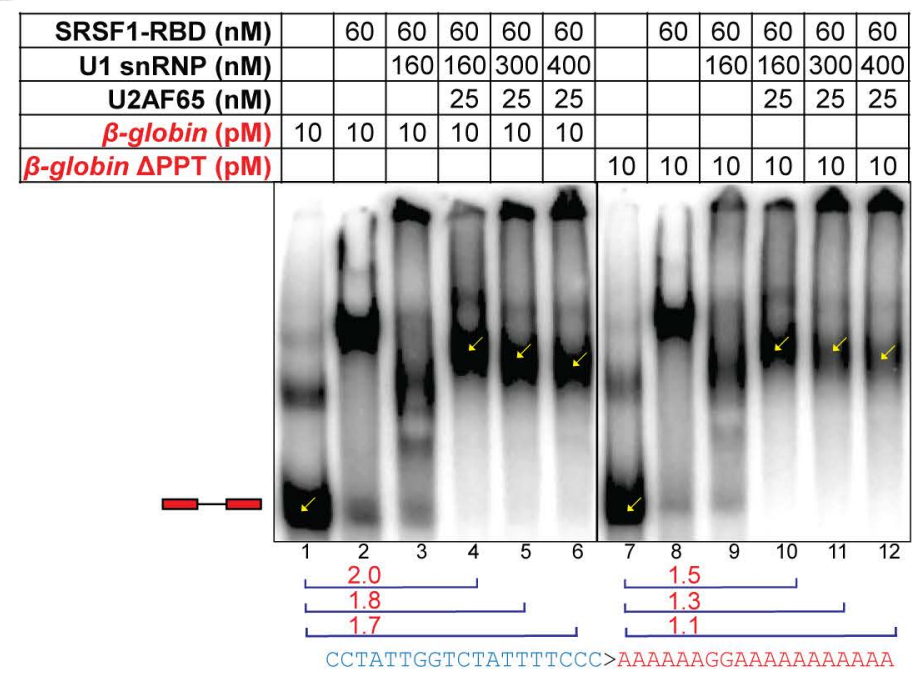

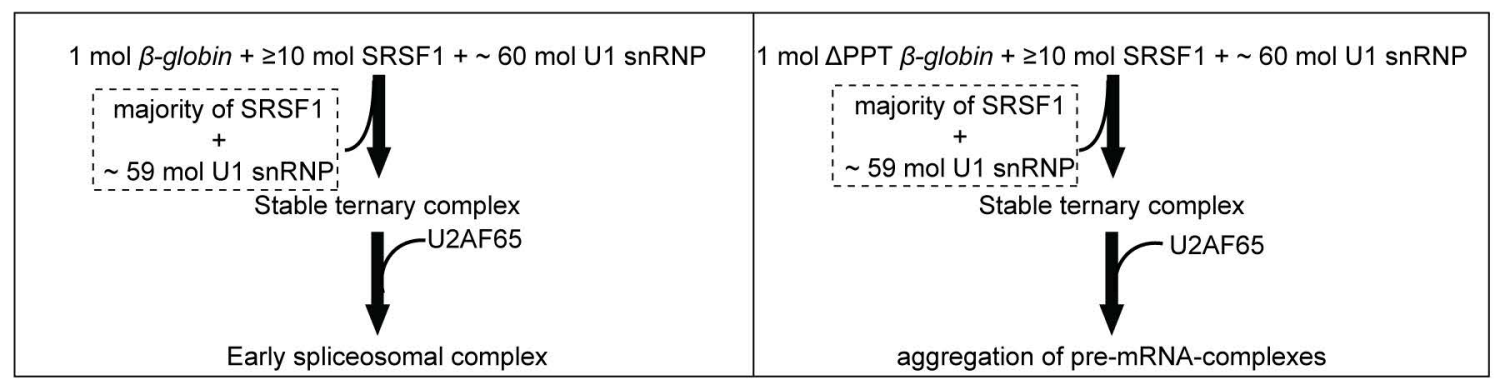




\section{A}

\begin{tabular}{|r|c|c|c|c|c|c|c|c|c|c|c|c|c|c|c|c|c|c|}
\hline SRSF1-RBD(nM) & & 550 & 250 & 200 & 150 & 75 & 50 & 150 & 75 & 50 & 150 & 75 & 50 & 150 & 75 & 50 & 150 & 75 \\
\hline SRSF2-RE (nM) & & & & & & & & 250 & 250 & 250 & 175 & 175 & 175 & 75 & 75 & 75 & 50 & 50 \\
\hline U1 snRNP (nM) & & & 1.5 & 1.5 & 1.5 & 1.5 & 1.5 & 1.5 & 1.5 & 1.5 & 1.5 & 1.5 & 1.5 & 1.5 & 1.5 & 1.5 & 1.5 & 1.5 \\
\hline B-globin (nM) & 25 & 25 & 25 & 25 & 25 & 25 & 25 & 25 & 25 & 25 & 25 & 25 & 25 & 25 & 25 & 25 & 25 & 25 \\
\hline
\end{tabular}

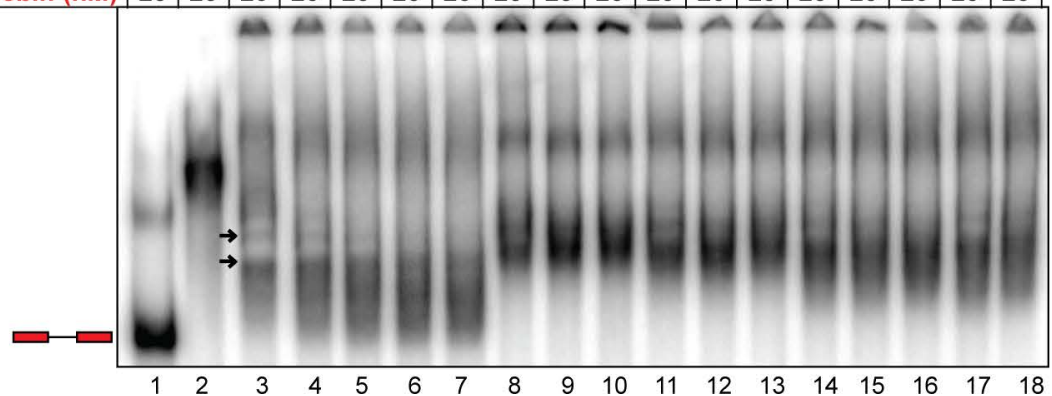

C

\begin{tabular}{|c|c|c|c|c|c|c|c|c|c|c|c|c|c|c|c|c|}
\hline SRSF1-RBD (nM) & & 20 & & 20 & & 20 & & 20 & & 20 & \multirow{2}{*}{$\begin{array}{c}\beta-g l o b i n \\
\text { variants }\end{array}$} & \multirow{2}{*}{5} & \multirow{2}{*}{$\begin{array}{l}0 \\
\infty \\
n \\
\text { D }\end{array}$} & \multirow{2}{*}{ 号 } & \multirow{2}{*}{$\frac{5}{\frac{n}{4}}$} & \multirow{2}{*}{\begin{tabular}{|l|} 
\\
का \\
m \\
\end{tabular}} \\
\hline SRSF2-RE (nM) & & 10 & & 10 & & 10 & & 10 & & 10 & & & & & & \\
\hline U1 sn & & 400 & & 400 & & 400 & & 400 & & 400 & & & & & & \\
\hline IIII) & & 25 & & 25 & & 25 & & 25 & & 25 & & & & & - & - \\
\hline$\beta$-globin (10 pM) & 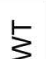 & 5 & & क & $\mathscr{m}$ & 芩 & & 占 & लD & m & & & & & & \\
\hline
\end{tabular}

$\triangle B S:$ CACTGAC>CGCTGGC $\triangle 3$ 'SS: $A G>C C$
Figure 4

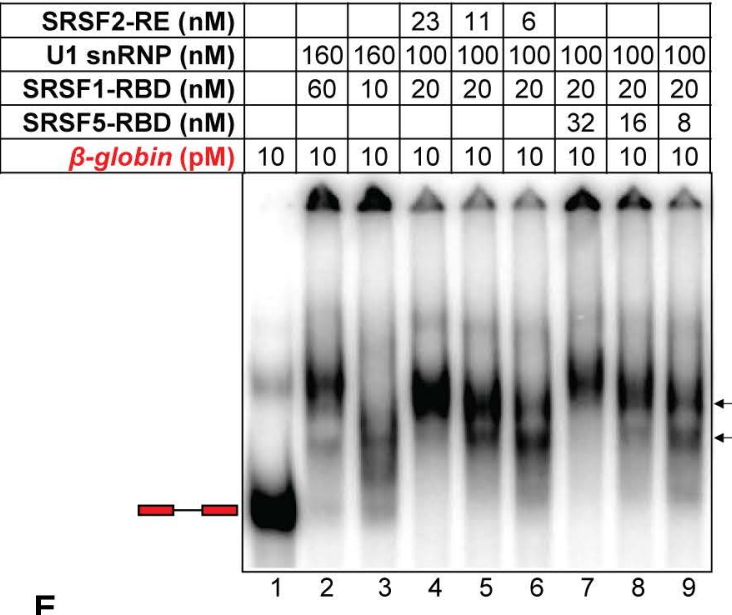

E

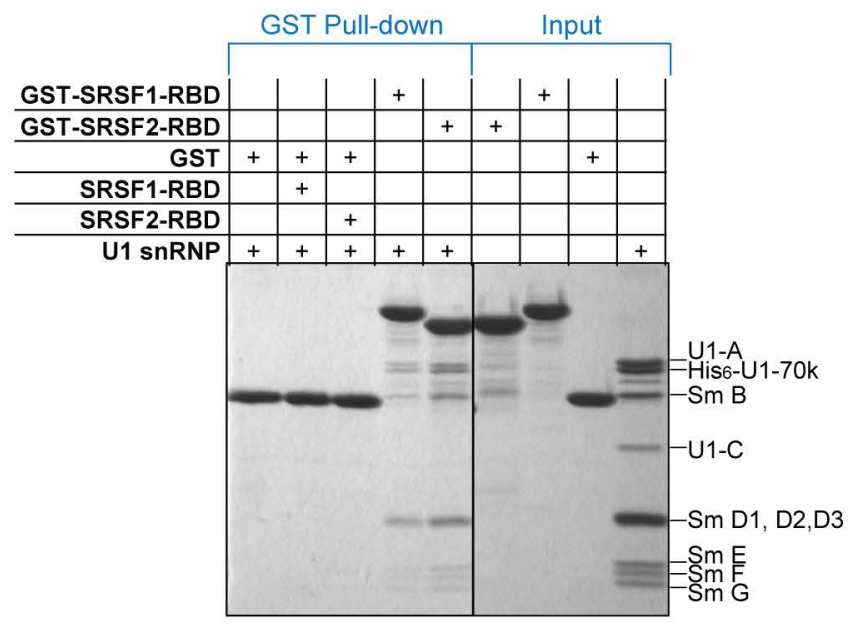

\section{F}

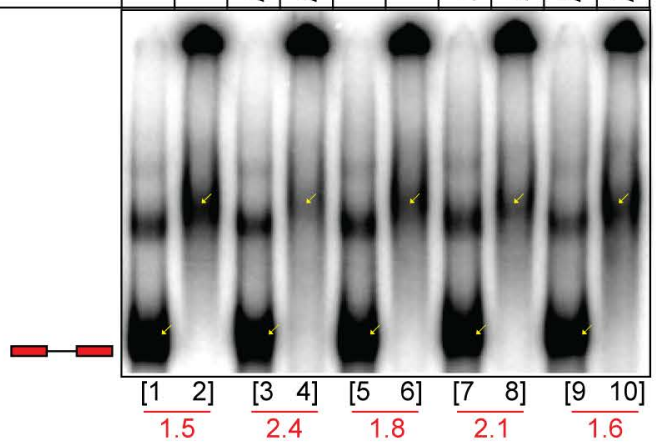

\begin{tabular}{|c|c|}
\hline $\begin{array}{r}1 \mathrm{~mol} \beta \text {-globin }+4 \mathrm{~mol} \text { SRSF1 }+6 \text { mol SRSF2 } \\
+4 \mathrm{~mol} \text { SRSF5 + } 40 \text { mol U1 snRNP }\end{array}$ & $\begin{array}{l}1 \text { mol } \beta \text {-globin splice signal mutant }+4 \text { mol SRSF1 } \\
+6 \text { mol SRSF2 }+4 \text { mol SRSF5 }+\sim 40 \text { mol U1 snRNP } \\
\text { Aggregate-like complexes }\end{array}$ \\
\hline
\end{tabular}



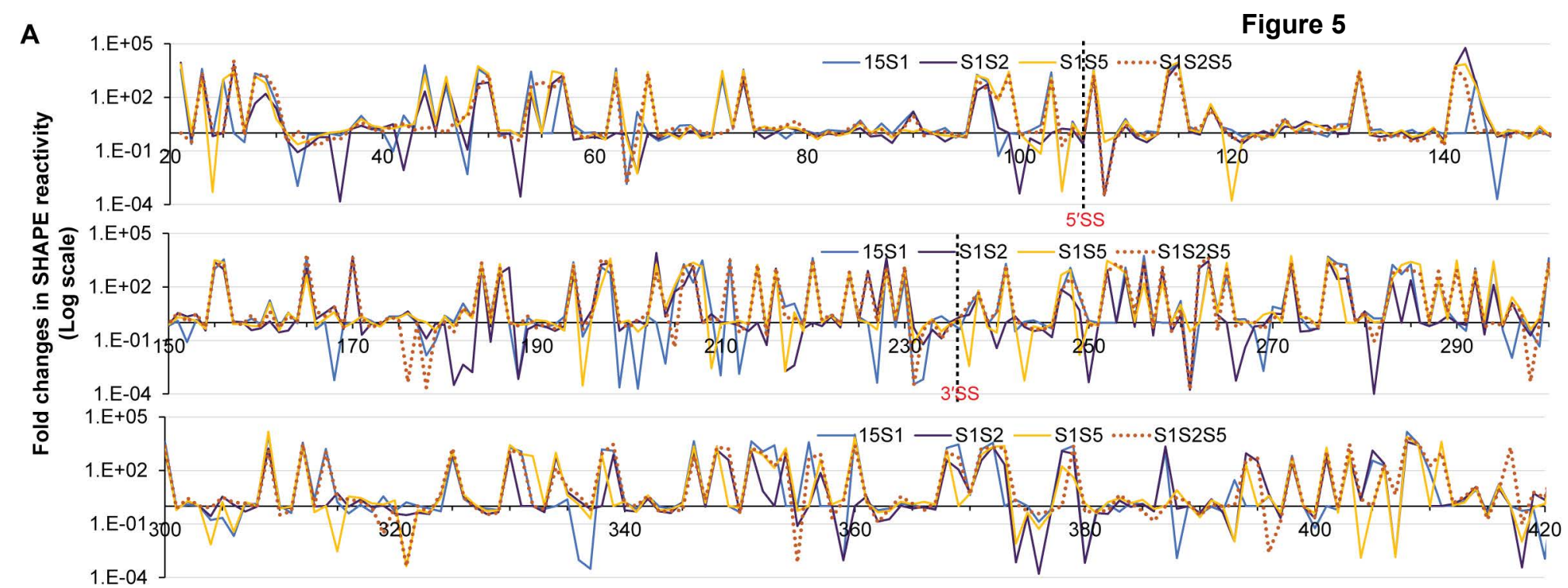

B
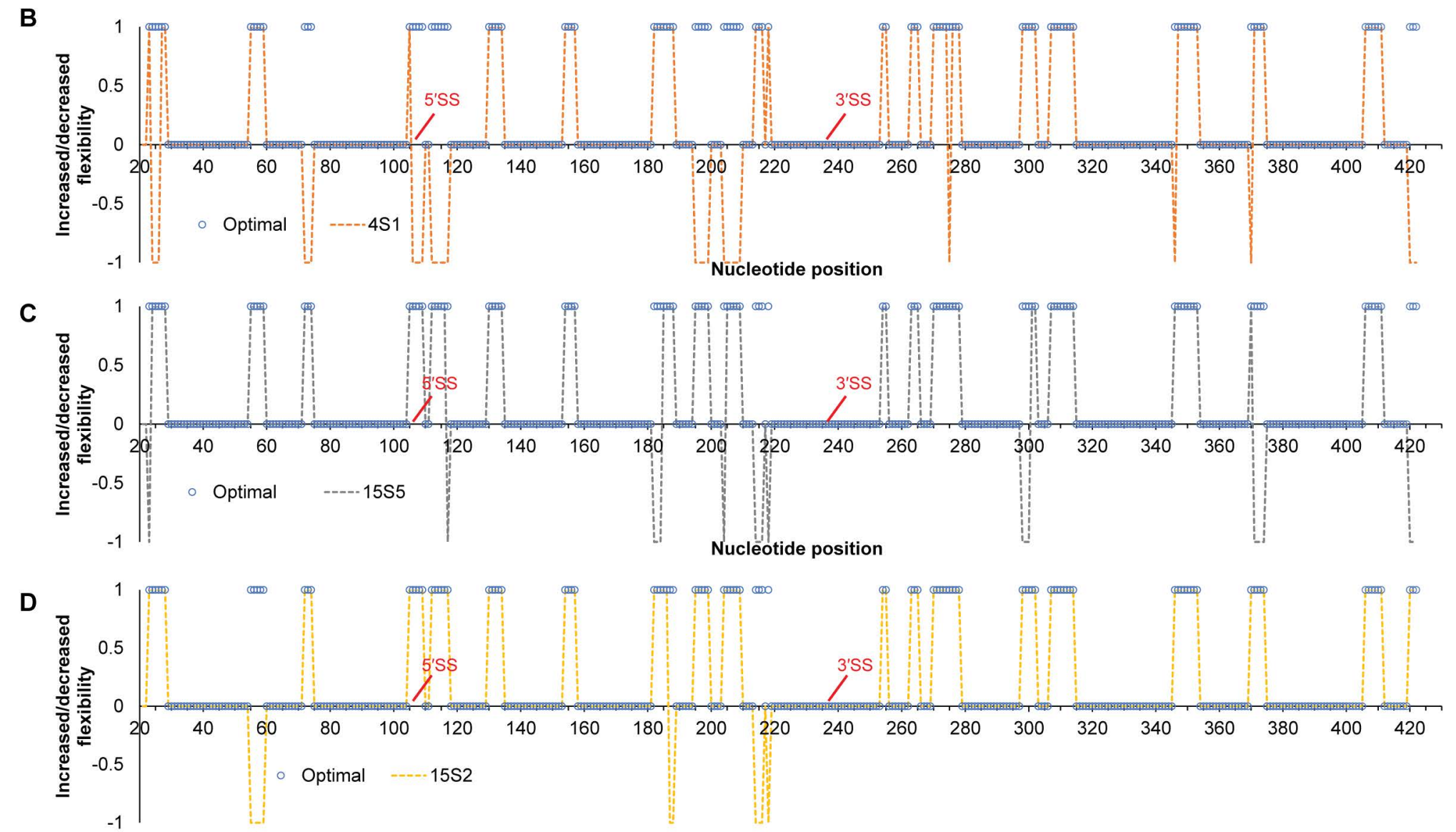


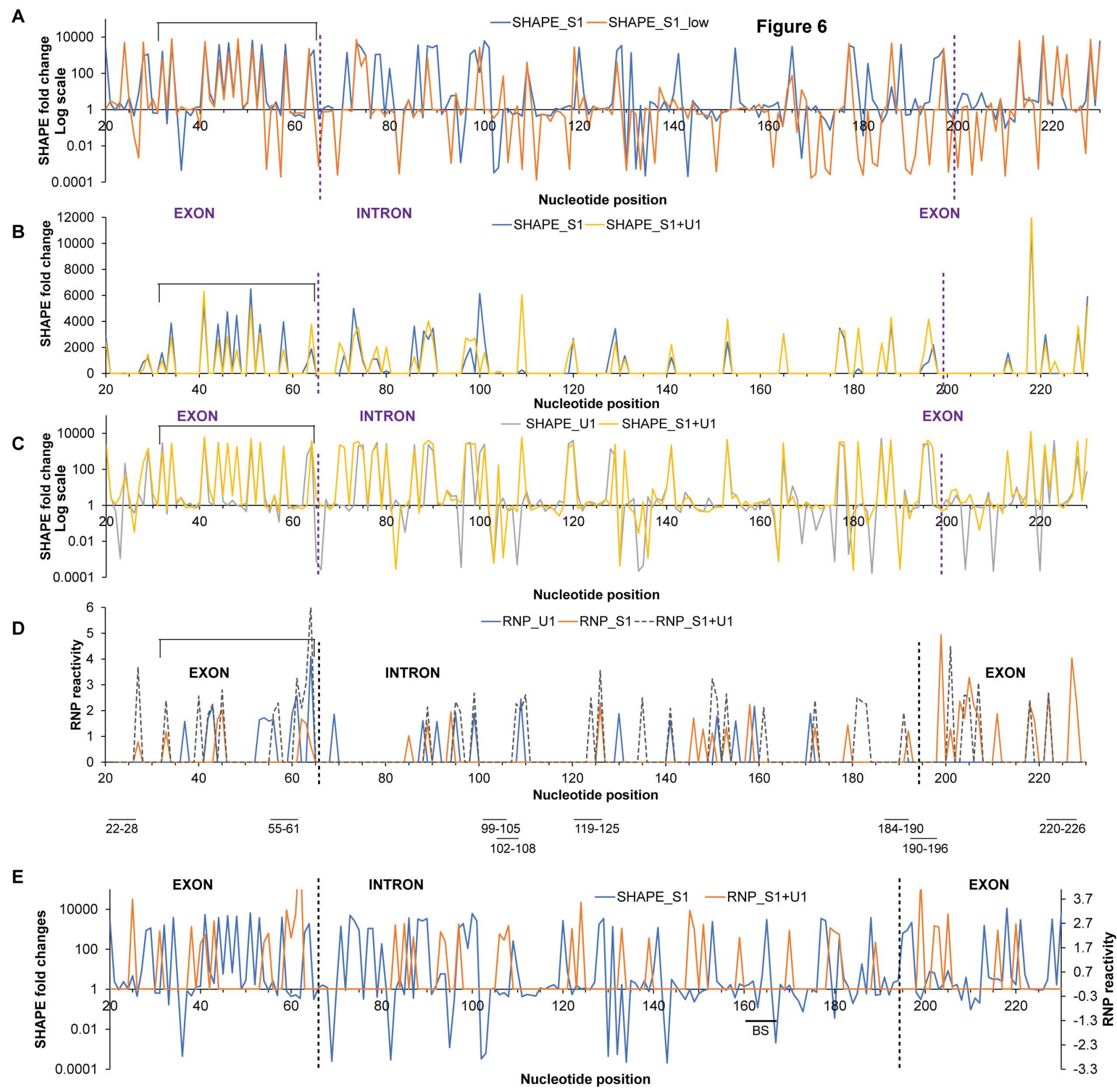




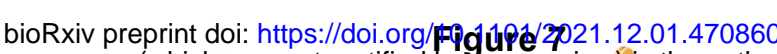
(which was not certified by peer review) is the autho
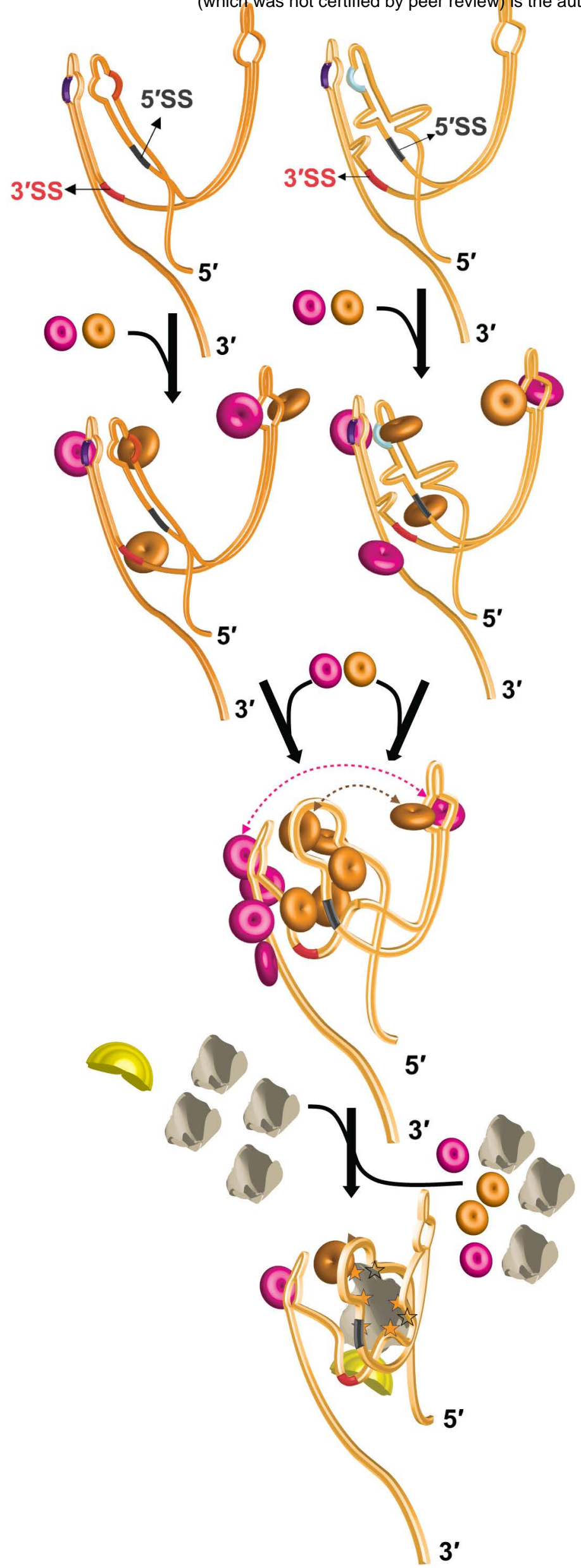

$=\mathrm{U} 1 \mathrm{snRNP}=\mathrm{U} 2 \mathrm{AF} 65$

\section{(0), = Splicing regulatory proteins (SRps)}

